\title{
FOXN1 compound heterozygous mutations cause selective thymic hypoplasia in humans
}

\author{
Qiumei Du, ${ }^{1}$ Larry K. Huynh, ${ }^{1}$ Fatma Coskun, ${ }^{1}$ Erika Molina, ${ }^{1}$ Matthew A. King, ${ }^{1}$ Prithvi Raj, ${ }^{1}$ Shaheen Khan, ${ }^{1}$ Igor Dozmorov, ${ }^{1}$ \\ Christine M. Seroogy, ${ }^{2}$ Christian A. Wysocki, ${ }^{3,4}$ Grace T. Padron, ${ }^{5}$ Tyler R. Yates, ${ }^{6}$ M. Louise Markert, ${ }^{6,7}$ M. Teresa de la Morena, ${ }^{8}$ \\ and Nicolai S.C. van Oers ${ }^{1,3,9}$ \\ 'Departments of Immunology, University of Texas Southwestern Medical Center, Dallas, Texas, USA. ${ }^{2}$ Department of Pediatrics, University of Wisconsin School of Medicine and Public Health, Madison, \\ Wisconsin, USA. ${ }^{3}$ Department of Pediatrics, and ${ }^{4}$ Department of Internal Medicine, University of Texas Southwestern Medical Center, Dallas, Texas, USA. ${ }^{5}$ University of Miami Miller School of Medicine, \\ Miami, Florida, USA. ${ }^{6}$ Department of Pediatrics and 'Department of Immunology, Duke University Medical Center, Durham, North Carolina, USA. ${ }^{8}$ Division of Immunology, Department of Pediatrics, \\ University of Washington and Seattle Children's Hospital, Seattle, Washington , USA. ${ }^{9}$ Department of Microbiology, University of Texas Southwestern Medical Center, Dallas, Texas, USA.
}

\begin{abstract}
We report on 2 patients with compound heterozygous mutations in forkhead box N1 (FOXN1), a transcription factor essential for thymic epithelial cell (TEC) differentiation. TECs are critical for T cell development. Both patients had a presentation consistent with $\mathrm{T}^{-/ / 10} \mathrm{~B}^{+} \mathrm{NK}^{+} \mathrm{SCID}$, with normal hair and nails, distinct from the classic nude/SCID phenotype in individuals with autosomal-recessive FOXN1 mutations. To understand the basis of this phenotype and the effects of the mutations on FOXN1, we generated mice using CRISPR-Cas9 technology to genocopy mutations in 1 of the patients. The mice with the Foxn1 compound heterozygous mutations had thymic hypoplasia, causing a $\mathrm{T}^{-} \mathrm{B}^{+} \mathrm{NK}^{+} \mathrm{SCID}$ phenotype, whereas the hair and nails of these mice were normal. Characterization of the functional changes due to the Foxn1 mutations revealed a 5-amino acid segment at the end of the DNA-binding domain essential for the development of TECs but not keratinocytes. The transcriptional activity of this Foxn1 mutant was partly retained, indicating a region that specifies TEC functions. Analysis of an additional 9 FOXN1 mutations identified in multiple unrelated patients revealed distinct functional consequences contingent on the impact of the mutation on the DNA-binding and transactivation domains of FOXN1.
\end{abstract}

\section{Introduction}

The development of $\mathrm{T}$ cells expressing $\mathrm{T}$ cell receptors (TCRs) bearing a precise specificity for peptides embedded in self-HLA (MHC) molecules occurs in the thymus. At this site, T cell interactions with thymic epithelial cells (TECs) establish an individualized TCR repertoire. The developmental progression of $\mathrm{T}$ cells occurs in several stages and involves 2 distinct epithelial cell (EC) subsets, cortical and medullary TECs $(1,2)$. Designated by their distribution within the thymus, these subsets are functionally distinct despite developing from a common precursor cell (3). Cortical TECs (cTECs) support early T cell development. They release chemokines such as $\mathrm{Ccl} 19, \mathrm{Ccl} 21$, and $\mathrm{Ccl} 25$ to direct early thymocyte precursor entry at a cortical-medullary interface (4). cTECs also secrete IL-7, and in conjunction with the chemokines, combinatorially provide proliferation, differentiation, and directional information to the immature double-negative (DN) $\mathrm{CD}^{-}{ }^{-} \mathrm{CD} 8$ thymocyte subset (4). This is further determined by the expression on cTECs of delta ligand-like 4 (DDL4), which binds to Notch on immature thymocytes and leads to expression of the Notch-acti-

Conflict of interest: MLM developed thymus transplantation intellectual property, which has been licensed to Enzyvant Therapeutics. Both MLM and Duke University may benefit financially if the technology is commercially successful in the future. Copyright: (5) 2019, American Society for Clinical Investigation.

Submitted: January 25, 2019; Accepted: August 1, 2019; Published: September 30, 2019 Reference information: / Clin Invest. 2019;129(11):4724-4738.

https://doi.org/10.1172/JCl127565. vated transcription factor RBPJк. The various cTEC-dependent signals promote chromosome rearrangements at the TCR $\beta$ locus in the DN thymocytes. Successful expression of a TCR $\beta$ protein signals an expansion and differentiation phase of the DN cells into the double-positive (DP) $\mathrm{CD} 4^{+} \mathrm{CD} 8^{+}$subset. During this transition, the TCR $\alpha$ locus is rearranged, with a productively produced TCR $\alpha$ protein pairing with the TCR $\beta$ subunit. A byproduct of this rearrangement event is the TCR excision circle (TREC), which is the newborn screening analyte used to identify patients with SCID (5).

The survival of an $\alpha \beta$ TCR-expressing DP thymocyte is enabled after favorable "weak" TCR interactions with self-peptides bound to MHC molecules presented by the cTECs (positive selection). The positively selected thymocytes are directed into the $\mathrm{CD}^{+} \mathrm{CD}^{-}$and $\mathrm{CD}^{-} \mathrm{CD}^{+}$single-positive (SP) subsets that enter the medullary regions. The expansion and function of medullary TECs (mTECs) relies on their crosstalk with SP thymocytes, with CD40L, RANKL, and EGF engaging CD40, RANK, and EGFR, respectively, on the TECs $(6,7)$. The mTECs present many tissue-restricted antigens on HLA molecules, a process that requires the transcriptional regulator termed AIRE, along with a second transcription factor, Fezf $2(8,9)$. mTECs purge T cells with high-affinity, self-reactive TCR specificities (negative selection). An added function of mTECs is to support the selection of Tregs (10). The selected Tregs exit into the peripheral lymphoid organs, where they prevent the promiscuous activation of any remaining autoreactive T cells (11). The gauntlet of cTEC- and mTECmediated $\mathrm{T}$ cell selection processes, aided by DCs, results in pro- 
Table 1. Clinical presentations of 2 patients identified with compound heterozygous FOXN1 mutations

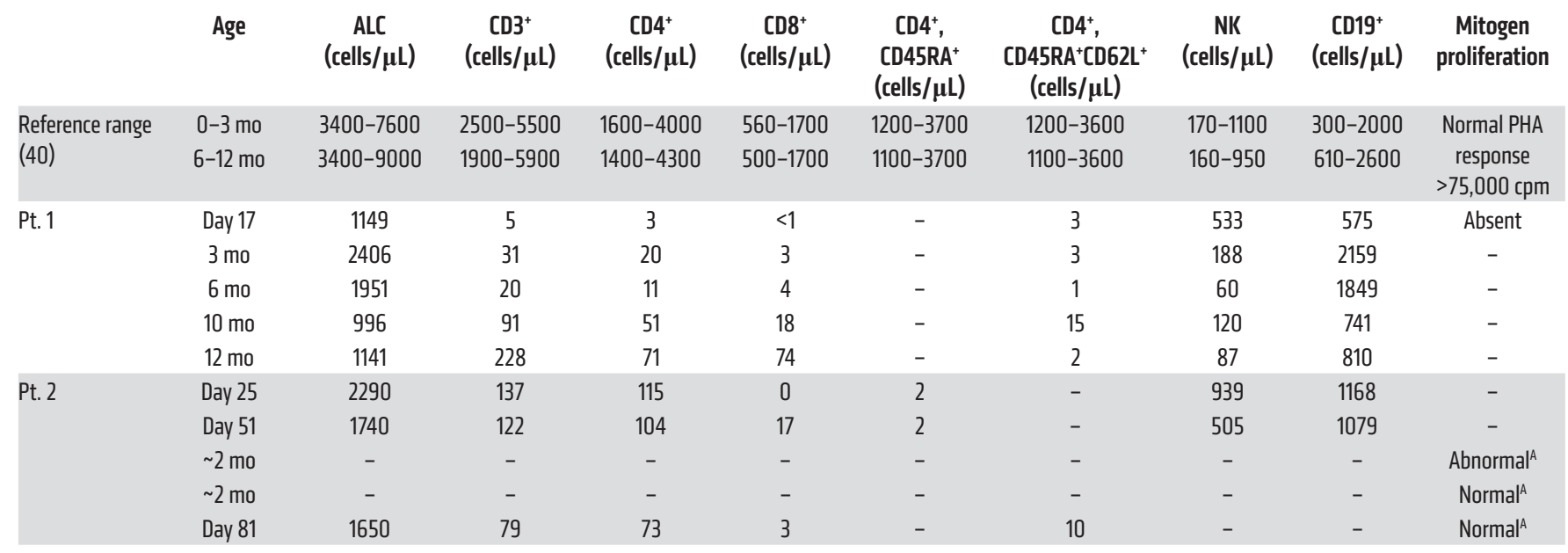

AThe proliferation assays were conducted in 3 different laboratories; the initial mitogen proliferation used anti-CD3, anti-CD28, and anti-IL-2 stimulation, whereas the subsequent 2 assays were performed using phytohemagglutinin stimulation at 2 separate laboratories. ALC, absolute lymphocyte count; PHA, phytohemagglutinin.

grammed cell death for the vast majority of thymocytes. What emerges from the thymus is a population of mature $\mathrm{T}$ cells with a precise TCR specificity unique to a given individual.

The development and differentiation of TECs into the cortical and medullary subsets are established by the transcription factor forkhead box N1 (FOXN1) $(1,12)$. FOXN1 belongs to a large family of proteins defined by a conserved, 100-amino-acid-long DNA-binding domain called the forkhead or winged helix. Forkhead family members are present in species from yeast to humans, but not in plants $(13,14) . F O X N 1$ arose via duplication of FOXN4, an ancestral homolog that first appeared in those early chordates with evidence of a thymus $(1,15,16)$. Comprising $3 \alpha$ helices, $3 \beta$ sheets, and 2 loops designated as the wings, the third $\alpha$ helix of FOXN1 contacts the major groove of DNA (17). The second winged segment provides additional binding specificity within the minor groove of DNA. Foxn1 interacts with DNA sequences containing a GAa/cGC consensus and transcriptionally activates nearly 500 genes including keratins, cytokeratins, proteasome components, and cell-surface proteins (2). A transactivation domain near the $\mathrm{COOH}$-terminal half of the protein is also required for Foxn1 functions (18).

The importance of FOXN1 in TEC and keratinocyte development was first uncovered in a spontaneously arising nude phenotype in mice $(n u / n u)$, which was later shown to be caused by autosomal-recessive mutations in this gene $(12,19)$. The loss of Foxn1 protein expression results in a TEC deficiency in combination with structural defects of the hair shaft and nail beds $(12,20,21)$. This is the basis of the $\mathrm{T}^{-} \mathrm{B}^{+} \mathrm{NK}^{+}$nude/SCID phenotype. Since the original description of nude mice in 1966 and later, of nude rats, 3 distinct autosomal-recessive mutations in human FOXN1 have been reported in approximately 10 patients, all of whom presented with similar nude/SCID phenotypes (22-26). This phenotype is also described in Birman cats (27). The classic clinical presentations for humans with autosomal-recessive FOXN1 mutations include a $\mathrm{T}^{-} \mathrm{B}^{+} \mathrm{NK}^{+} \mathrm{SCID}$ phenotype due to thymic aplasia in conjunction with alopecia universalis and nail plate dystrophy $(23,25,28)$. Thymic hypoplasia/aplasia arising from defects with stromal cell populations such as TECs can also occur in individuals with mutations in CHD7, PAX1, TBX1, and TBX2, and in those with chromosome 22q11.2 deletion syndrome (DiGeorge syndrome) (29-35). However, only the autosomal-recessive mutations described for FOXN1 lead to the additional developmental problems of the hair shaft and nail plate.

We report here on the identification of mutations in FOXN1 in 2 patients with a $\mathrm{T}^{-/ 1} \mathrm{~B}^{+} \mathrm{NK}^{+}$SCID without evidence of alopecia or nail dystrophy. We used CRISPR-Cas9 technology to develop Foxn1 compound heterozygous mice that genocopied selected human FOXN1 mutations. A comparison of the various mice harboring homozygous and compound heterozygous Foxn1 mutations revealed differential functions of this transcription factor in TECs versus keratinocytes. Phenotype analyses and functional assays revealed an important 5-amino acid sequence in Foxn1 that differentiates its role in TECs versus keratinocytes. A comparative analysis of 12 distinct FOXN1 mutations, identified in patient 1 (Pt. 1) and patient 2 (Pt. 2) as well as in several unrelated patients with heterozygous mutations, revealed varying transcriptional activities depending on the location and type of mutation. These findings provide structure-function insights into this key transcription factor.

\section{Results}

Compound heterozygous mutations in FOXN1 identified in 2 unrelated patients with a SCID phenotype without alopecia. Pt. 1 was a female born at full term to nonconsanguineous parents and was identified as having SCID following newborn screening that revealed undetectable TRECs. The subsequent clinical work-up was consistent with a typical $\mathrm{T}^{-} \mathrm{B}^{+} \mathrm{NK}^{+}$SCID phenotype, using the normal age-matched reference range (Table 1) $(36,37)$. Exome sequencing revealed 2 mutations in the FOXN1 gene, a duplication 


\section{Table 2. Clinical presentations of patients with compound heterozygous or monoallelic mutations in FOXN1}

\begin{tabular}{|c|c|c|c|c|c|c|c|}
\hline Patient & DNA mutation & $\begin{array}{l}\text { Protein-coding } \\
\text { mutation }\end{array}$ & $\begin{array}{l}\text { Protein function (\% of } \\
\text { WT) (luciferase assay) }\end{array}$ & $\begin{array}{l}\text { Abnormal } \\
\text { hair }\end{array}$ & $\begin{array}{l}\text { Abnormal } \\
\text { nails }\end{array}$ & Transplant & Other clinical notes \\
\hline Pt. 1 & c.933_936dupACCC & p.T313fsX169 & $1.5 \%$ & No & No & No & $\begin{array}{l}\text { Multiple viral infections, failure to thrive. Died at } 1 \text { yr of age } \\
\text { from parainfluenza virus. }\end{array}$ \\
\hline Pt. 1 & c.1089_1103del15 & p.W363C & $31 \%$ & No & No & No & $\begin{array}{l}\text { Multiple viral infections, failure to thrive. Died at } 1 \text { yr of age } \\
\text { from parainfluenza virus. }\end{array}$ \\
\hline Pt. 2 & c. $1288 C>T$ & p.P430S & $100 \%$ & No & No & Yes & $\begin{array}{c}\text { Received BM transplant. Healthy, no recurrent infections, } \\
\text { on s.c. gamma globulin therapy. }\end{array}$ \\
\hline Pt. 3 & c.1465delC & p.R489fsX61 & $18 \%$ & No & No & No & Healthy, no recurrent infections. \\
\hline Pt. 4 & c.1465delC & p.R489fsX61 & $18 \%$ & Yes & No & No & Healthy, no recurrent infections. \\
\hline Pt. 5 & $724[>T$ & p.P242S & $95 \%$ & No & No & No & $\begin{array}{c}\text { Developed S. aureus submandibular abscess in infancy; } \\
\text { otherwise healthy. }\end{array}$ \\
\hline Pt. 9 & $1075 G>A$ & p.Е359K & $63 \%$ & No & No & No & Healthy, no recurrent infections. \\
\hline Pt. 10 & 1201_1206del16 & p.P401AfsX144 & $3 \%$ & No & No & No & $\begin{array}{l}\text { Also had heterozygous DOCK8 and CHD7 mutations } \\
\text { with no congenital anomalies. }\end{array}$ \\
\hline Pt. 11 & 1201_1206del16 & p.P401AfsX144 & $3 \%$ & No & No & No & Healthy, no recurrent infections. \\
\hline Pt. 12 & 1201_1206del16 & p.P401AfsX144 & $3 \%$ & No & No & No & Healthy, no recurrent infections. Received live virus vaccines. \\
\hline Pt. 13 & 1201_1206del16 & p.P401AfsX144 & $3 \%$ & No & Yes & No & Healthy, no recurrent infections. \\
\hline Pt. 14 & 1201_1206del16 & p.P401AfsX144 & $3 \%$ & No & No & No & $\begin{array}{l}\text { Born prematurely due to maternal infection. } \\
\text { Currently healthy with no recurrent infections. }\end{array}$ \\
\hline Pt. 15 & 1293delC & p.P432fsX118 & $2 \%$ & No & No & Yes & Low numbers through 3 mo when alternate therapy was given. \\
\hline Pt. 16 & 1418delC & p.P473HfsX77 & $12 \%$ & Yes & Yes & Yes & Low numbers through $\sim 4$ mo when alternative therapy was given. \\
\hline
\end{tabular}

S. aureus, Staphylococcus aureus.

of ACCC at position c.933 (c.933_936dup), and a 15-nt deletion (c.1089_1103del) (Table 2). Analysis of the parental data indicated that the 933 mutation was inherited from the mother, whereas the 1089 mutation was passed down from the father. Both parents were healthy and had no evidence of hair loss or nail changes (Figure 1, A, C, and D; Family 1). The c.933 duplication was identified as a presumed pathogenic variant that was predicted to result in a premature stop codon following a new coding sequence of approximately 169 amino acids extending from the middle of the DNA-binding domain (p.Thr313fs ${ }^{\star} 169$ ). The second allele in Pt. 1 contained a 15-nt deletion near the end of the DNA-binding domain. This resulted in a single amino acid conversion (Trp to Cys) followed by a 5-amino acid deletion (p.Trp363_Pro367delinsCys) (Figure 1, A and C). The deletion did not change the reading frame. The clinical course for Pt. 1 was characterized by rhino/ enteroviral infection at 2 months of age, norovirus enteritis and failure to thrive starting at 5 months, and parainfluenza virus type 3 (PIV3) at 9 months (Table 2). Although this infant was a candidate for allogeneic thymic tissue transplantation, the recurring viral infections resulted in her death before 1 year of age. This was a consequence of respiratory failure with disseminated PIV3, with identification of the virus also in liver biopsies.

Pt. 2, born to nonconsanguineous parents, also had undetectable TRECs following newborn screening. Serial immune testing was consistent with a $\mathrm{T}^{-/ 1} \mathrm{~B}^{+} \mathrm{NK}^{+}$leaky SCID phenotype with persistent severe $\mathrm{T}$ cell lymphopenia, absent thymopoiesis, and diminished $\mathrm{T}$ cell function (Table 1). This child underwent hematopoietic cell transplantation at 6 months of age (Table 2). Exome sequencing revealed c.C1288T and c.1465delC FOXN1 variants (Figure 1, B and E; family 2). Sanger sequencing confirmed individual FOXN1 allelic mutations in Pt. 2. The nucleotide changes caused a p.Pro430Ser on FOXN1 derived from 1 allele and a p.Gln489Argfs on the second allele that resulted in a frameshift mutation in the protein (Figure 1, B, C, and E). The 1288 variant is a polymorphism found in $4 \%$ of the general population (dbSNP: rs61749867), but the impact of this variant on FOXN1 has not been established. Analysis of the parents indicated that the c.C1288T variant was inherited from the mother, whereas the c.1465delC variant was de novo.

The FOXN1 mutations described for both Pt. 1 and Pt. 2 are distinct from the classic autosomal-recessive mutations originally identified in FOXN1 (Figure 1C) (22-26). To date, previously identified mutations on other genes associated with SCID or cellular immunodeficiency phenotypes were not found in Pt. 1 or Pt. 2 (Table 2).

Mice harboring compound heterozygous mutations in Foxn1 have thymic hypoplasia with normal fur and nail beds. To confirm that the FOXN1 mutations in Pt. 1 were causal to the SCID phenotype, we used a CRISPR-Cas9 strategy to create analogous mutations in $\mathrm{C} 57 \mathrm{Bl} / 6$ mice. Individual guide RNAs were designed with Cas9 cleavage sites near the equivalent positions in the murine locus 
A

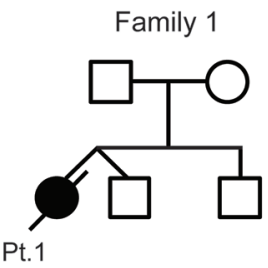

C FOXN1 CDNA
B Family 2

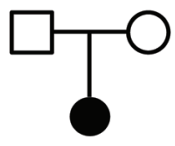

Pt. 2

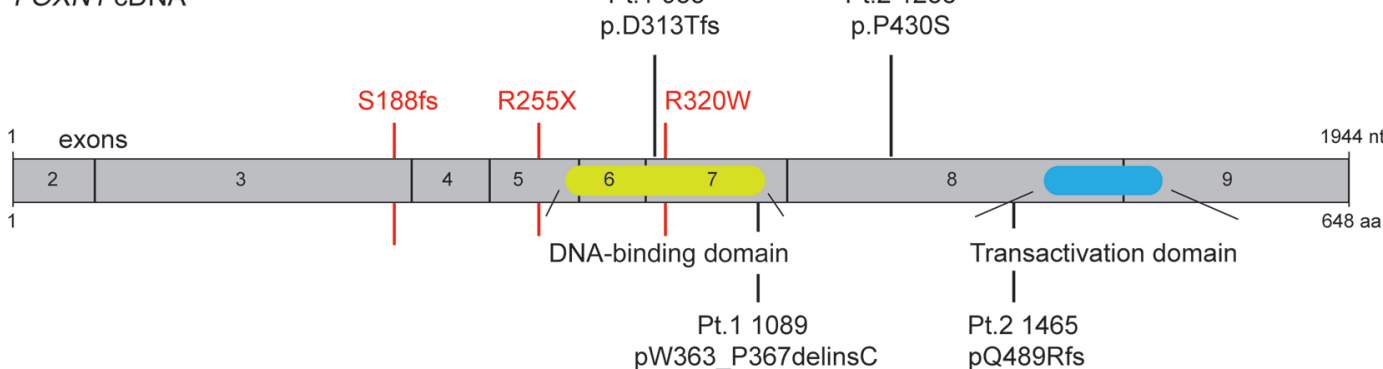

\begin{tabular}{|c|c|c|c|c|}
\hline D & Pt.1 & 33 4-nt duplication ( $1^{\text {st }}$ allele) & Pt.1 & 089 15-nt deletion ( $2^{\text {nd }}$ al \\
\hline DNA & $\begin{array}{l}\text { WT } \\
\text { Pt.1 }\end{array}$ & $\begin{array}{l}\text { GACAGCACCCGATGGCTG } \\
\text { GACAGCACCCACCCCGATGGCTG }\end{array}$ & $\begin{array}{l}\text { WT } \\
\text { Pt.1 }\end{array}$ & $\begin{array}{l}\text { CAAAAATGGAAGAGGA } \\
\text { CAAAAATG }\end{array}$ \\
\hline Protein & $\begin{array}{l}\text { WT } \\
\text { Pt.1 }\end{array}$ & $\begin{array}{l}\text { TAPDGWK } \\
\text { TAPT fs } 169\end{array}$ & $\begin{array}{l}\text { WT } \\
\text { Pt.1 }\end{array}$ & $\begin{array}{l}\text { QKWKRKDPIAV } \\
\text { QKC IAV }\end{array}$ \\
\hline & Pt.2 & $288 \mathrm{C} / \mathrm{T}$ mutation ( $1^{\text {st }}$ allele) & Pt.2 & $465 \mathrm{C}$ deletion $\left(2^{\text {nd }}\right.$ all \\
\hline DNA & $\begin{array}{l}\text { WT } \\
\text { Pt.2 }\end{array}$ & $\begin{array}{l}\text { CACCCAGCTCCAGGCCCC } \\
\text { CACCCAGCTTCAGGCCCC }\end{array}$ & $\begin{array}{l}\text { WT } \\
\text { Pt.2 }\end{array}$ & $\begin{array}{l}\text { ACCCCCCCAGGACTCC } \\
\text { ACCCCC_AGGACTCC }\end{array}$ \\
\hline Protein & $\begin{array}{l}\text { WT } \\
\text { Pt.2 }\end{array}$ & $\begin{array}{l}\text { HPAPGPIAG } \\
\text { HPAPGSIAG }\end{array}$ & $\begin{array}{l}\text { WT } \\
\text { Pt.2 }\end{array}$ & $\begin{array}{l}\text { TPQDSPLP } \\
\text { TPR fSX61 }\end{array}$ \\
\hline
\end{tabular}

Figure 1. Compound heterozygous mutations in FOXN1 identified in 2 patients. (A and B) Pt. 1 and Pt. 2 were identified from 2 independent and unrelated families. (C) Domain structure of FOXN1, with the 2 domains characterized to date: a DNA-binding region in the middle of the protein and a transactivation domain near the $\mathrm{COOH}$ terminus. Three FOXN1 autosomal-recessive mutations previously reported in patients with nude/SCID phenotypes are listed in red. Two patients, Pt. 1 and Pt. 2, presented with compound heterozygous mutations in FOXN1 at distinct sites, which are indicated above the exon assembly. ( $\mathbf{D}$ and $\mathbf{E}$ ) The DNA sequence mutations in FOXN1 and the corresponding effects on the amino acid sequence are shown for Pt. 1 (D) and Pt. 2 (E). The amino acid changes resulting from the various FOXN1 mutations are illustrated.

for Pt. 1, at positions 933 and 1089 (Figure 2A). Silent mutations were included to create new DNA restriction sites for genotyping purposes. The more difficult design was for position 933 (Pt. 1). At this location, the 4-nt duplication (ACCC) in human FOXN1 creates a frameshift that begins mid-way through the DNA-binding domain. The corresponding TCCT insertion within the same location in the murine Foxn1 locus creates an immediate stop codon. Consequently, a TCCC insertion was engineered into the murine locus along with several additional nucleotide substitutions within the repair template to better mimic the read-through seen with the patient's allele. The resulting coding sequences for both the human and murine genes following the insertion site were novel, with a new stop codon introduced within exon 6 . The corresponding mutations in the human genome led to a longer transcript, again with codons unrelated to WT FOXN1.

The phenotypes of the founder pups derived from the CRISPRCas9 genome editing of the Foxn1 933-nt position were distinct from those targeted at position 1089. Many of the 933 pups had a nude, runted phenotype (Supplemental Figure 1A; supple- mental material available online with this article; https://doi. org/10.1172/JCI127565DS1). DNA sequencing of these runts revealed deletions within the DNA-binding domain on both Foxn1 alleles. In contrast, the 1089 founders appeared visually indistinguishable from normal mice (Figure 2B and Supplemental Figure 1A). Founders with the correct nucleotide duplication (933 nt) or deletion (1089 nt) in Foxn1 were bred with WT mice, followed by intercrossing of the mice to establish the appropriate homozygous and compound heterozygous lines.

F2-generation mice homozygous for the 933 mutation (Foxn1 ${ }^{933 / 933}$ ) were small and hairless and had diminished nail lengths and shortened whiskers compared with littermate controls (Figure 2B and Supplemental Figure 1B). These phenotypes matched that of the classic $n u / n u$ mouse (12). In contrast, the physical appearance of the Foxn $1^{1089 / 1089}$ and Foxn $1^{933 / 1089}$ mice matched that of their littermate controls (Figure 2B). The Foxn $1^{933 / 933}$ mice had thymic aplasia, with a 35- to 50-fold reduction in thymus weights compared with littermate control thymus weights (Figure 2, C and D). The Foxn $1^{1089 / 1089}$ and Foxn1 $1^{933 / 1089}$ compound hetero- 


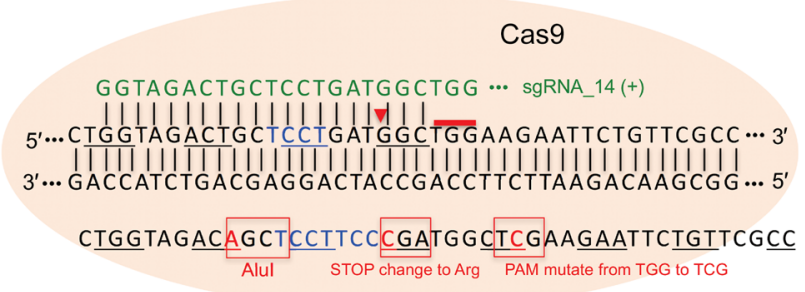

Foxn1 murine sequence

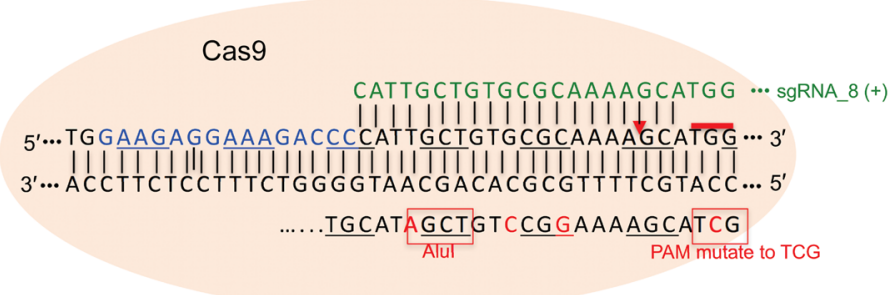

Foxn1 murine sequence

\section{B}
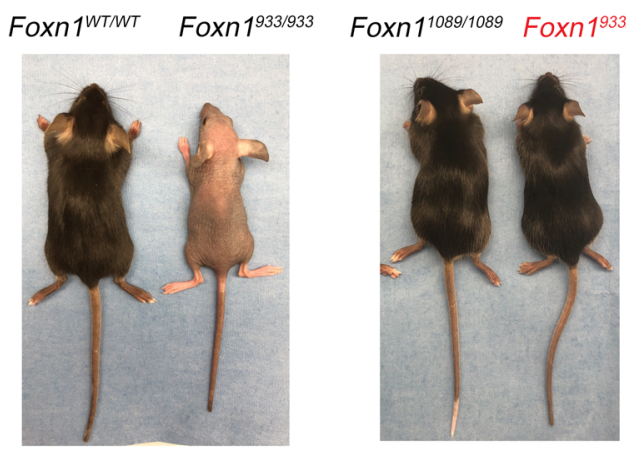

C

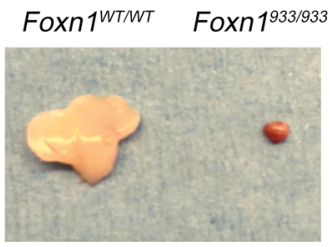

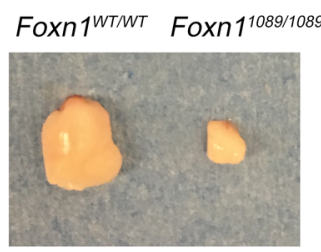

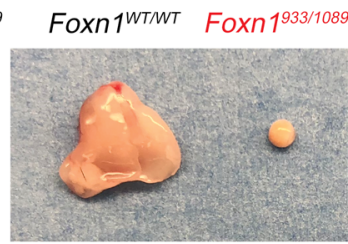

D

Thymus weights and cellularity
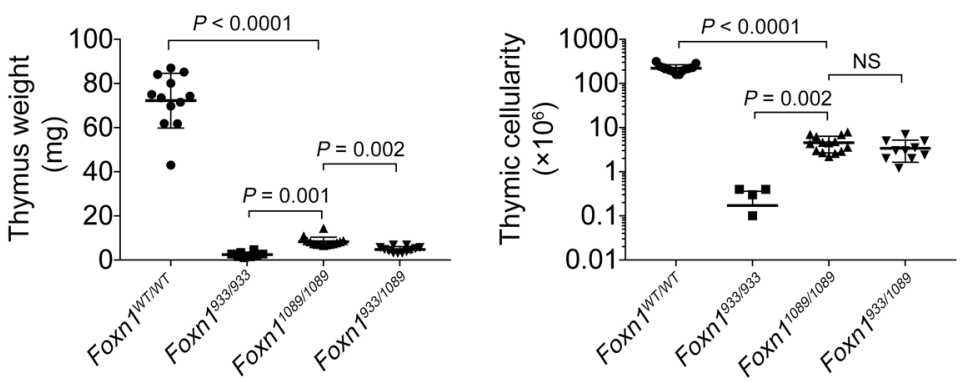

Figure 2. Human FOXN1 compound heterozygous mutations genocopied in mice cause thymic aplasia with normal fur and nails. (A) The human FOXN1 mutations for Pt. 1 were introduced into the murine genome by CRISPR-Cas9 technology. The DNA repair template used for each allele is shown. Silent mutations were introduced into the murine sequence to facilitate genotyping and to prevent premature stop codons. (B) Images of F2-generation mice:

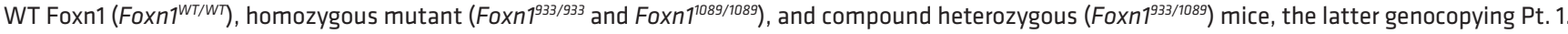
The genocopied mice are indicated in red font. The images are representative of 5 independently characterized mice. (C) The overall sizes of the thymi from the various mouse lines are shown for comparative purposes. (D) Thymus weights and overall thymic cellularity were calculated. Data represent the mean \pm SEM. $n=22$ Foxn $7^{W T / W T}, n=6$ Foxn $1^{933 / 933}, n=10$ Foxn 1089/1089, and $n=10$ Foxn1933/1089 mice. $P$ values of less than 0.05 were considered significant. For the comparisons shown, a Brown-Forsythe and Welch's 1-way ANOVA was applied.

zygous mice had pronounced thymic hypoplasia, with a 10- and 17-fold reduction in thymus weights, respectively (Figure 2, C and D). This was confirmed when we compared the total thymic cellularity of 6 to 22 mice per group (Figure 2D). Sex was not a determinant for the hypoplasia, as males and females were equally affected (Supplemental Figure 2A). A comparison of the various heterozygous mice derived from the intercrosses (Foxn $1^{\text {WT/1089 }}$ and Foxn1 ${ }^{\text {T/933 }}$ ) revealed no impact of a single-allele mutation on thymic cellularity, T cell development, and/or peripheral lymphocytes compared with littermate controls (Supplemental Figure 2, B-E). These findings firmly establish that the clinical phenotypes of Pt. 1 resulted from compound heterozygous mutations in FOXN1.

Thymopoiesis is severely attenuated in mouse lines with compound heterozygous mutations in Foxn1 that genocopy Pt. 1. To understand how the diverse FOXN1 mutations affect thymopoiesis, we compared the developmental progression of thymocytes in the different mouse lines (Figure 3A). We monitored the various stages by comparing the cell-surface expression of the CD4, CD8, TCR $\beta$, CD25, CD44, CD45, and CD69 by flow cytometry. As shown in the littermate controls (Foxn1 ${ }^{W T / W T}$ ), thymopoiesis was characterized by a subset of immature CD4-CD8 ${ }^{-}$cells $(\mathrm{DN}, 4 \%)$ that developed into a $\mathrm{CD} 4^{+} \mathrm{CD} 8^{+}$cell population (DP, $80 \%-87 \%$ ), of which a small number were positively selected into the $\mathrm{CD} 4^{+} \mathrm{CD} 8^{-}$(CD4 SP, 3\%-8\%) and CD4-CD8 ${ }^{+}$lineages (CD8 SP, 2\%-5\%) (Figure $3 \mathrm{~A})$. With the exception of some DN and CD8 ${ }^{10}$ cells, the DP and SP cell populations were virtually nonexistent in the Foxn1933/933 mouse line (Figure $3 \mathrm{~A})$. The $\mathrm{DN}$ and $\mathrm{CD} 8^{\text {lo }}$ cell populations lacked $\mathrm{CD} 45^{+}$cell-surface expression, indicating that they were not thy- 
A
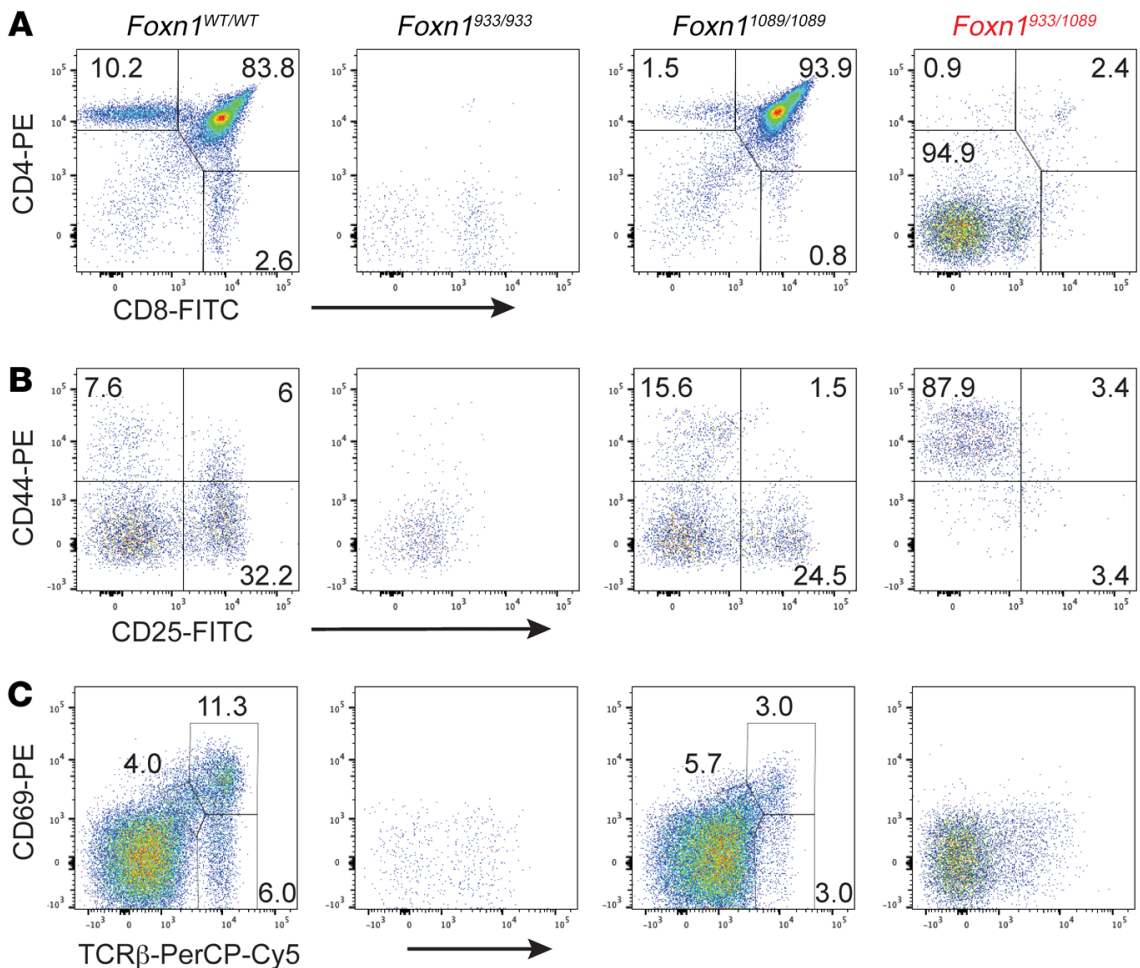

D
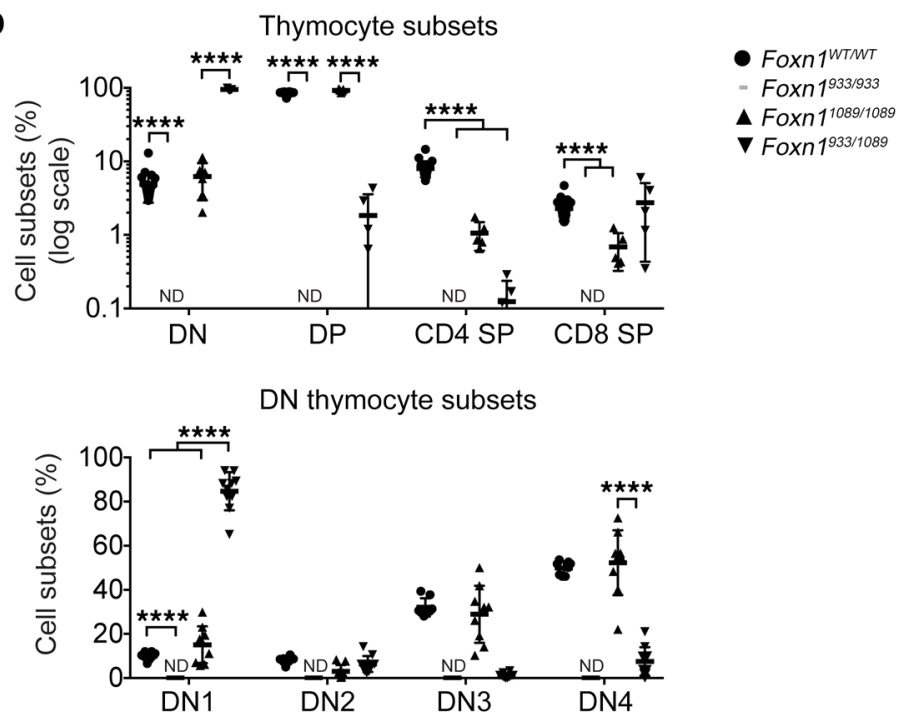

mocytes (Figure 3A, data not shown). This finding concurs with the cystic thymic rudiment described for the $n u / n u$ mice, which was devoid of thymocytes (38). The Foxn $1^{1089 / 1089}$ homozygous knockin mice had an increased percentage of DP thymocytes (90\%-95\%) with severely reduced SP subsets compared with controls. Such results suggest that homozygous mutations at the 1089 position of Foxn1 lead to some differentiation of thymocytes to the $\mathrm{CD} 4^{+} \mathrm{CD} 8^{+}$stage, at which point there was a developmental block. Thymopoiesis in the Foxn $1^{933 / 1089}$ mice, genocopying Pt. 1, was more severely affected. A significant reduction in the percentage of both DP and SP subsets was obvious, albeit not as extreme as that seen in the Foxn $1^{933 / 933}$ mouse line, a finding consistent with the presence of a small thymic structure (Figure 2C). These data confirm that the distinct Foxn 1 mutations have dif-
Figure 3. Thymic hypoplasia results from compound heterozygous mutations in Foxn1. (A-D) WT Foxn1 (Foxn1 ${ }^{W T / W T}$ ), homozygous mutant (Foxn1933/933, Foxn11089/1089), and compound heterozygous (Foxn $1^{933 / 1089}$ ) mice, the latter genocopying Pt. 1, were obtained by intercrossing the Foxn $7^{933 / W T}$ and Foxn 1089/WT lines. The thymus was isolated and single-cell suspensions prepared for flow cytometry. (A) Fluorochrome-labeled antibodies against CD4 and CD8 were used to detect DN, DP, and SP thymocyte subsets. (B) The progression of thymocytes from the DN1-DN4 stages of thymocytes was assessed by cell-surface staining for CD44 and CD25 following exclusion of cells expressing CD3, CD4, CD8, B220, NK1.1, TCR $\gamma \delta$, Ter199, CD11b, and CD11c. (C) Positive selection of thymocytes was assessed by staining thymocytes for TCR $\beta$ and CD69. (D) Graphs show the percentage of various cell subsets, determined from pooled experiments using a minimum of 5 mice per line. Data indicate the mean \pm SEM. $n=22$ Foxn $7^{W T / W T}$ mice, $n=6$ Foxn $1^{933 / 933)}$ mice, $n=6$ Foxn $1^{1089 / 1089}$ mice, and $n=$ 5 Foxn $7^{933 / 1089}$ mice. For the comparisons shown, a Brown-Forsythe and Welch's 1-way ANOVA was applied. $P$ values of less than 0.05 were considered significant. ${ }^{* * *} P<0.0001$ for most comparisons, with the following exceptions: $P=0.002$ for $D N$ comparisons of Foxn $1^{933 / 933}$ versus Foxn1089/1089; $P=$ NS for DP comparisons of Foxn ${ }^{W T / W T}$ versus Foxn $1^{1089 / 1089}$ and Foxn $1^{933 / 933}$ versus Foxn $1^{933 / 1089} ; P$ $=0.002$ for CD4 ${ }^{+}$SP Foxn $1^{933 / 933}$ versus Foxn $1^{1089 / 1089}$ and Foxn $11^{1089 / 1089}$ versus Foxn $1^{933 / 1089} ; P=$ NS for Foxn $1^{933 / 933}$ versus Foxn $1^{933 / 1089} ; P=0.05$ for CD8 ${ }^{+}$ SP Foxn $1^{933 / 933}$ versus Foxn $1^{933 / 1089}$. Comparisons for DN1-DN4 were as follows: $P=$ NS for DN1 differences between Foxn $1^{\text {WT/WT }}$ and Foxn 1089/1089;

$P=0.008$ for DN2 comparisons of Foxn $7^{W T / W T}$ versus Foxn1 ${ }^{1089 / 1089}, P=0.002$ for DN2 comparisons of Foxn1 $1^{933 / 933}$ versus Foxn1 $1^{1089 / 1089}$, and $P=$ NS for DN2 comparisons of Foxn 1089/1089 versus Foxn $1^{933 / 1089}$; $P$ $=0.002$ for DN3 comparisons of Foxn $7^{933 / 933}$ versus Foxn1 $1^{1089 / 1089}$ and $P=$ NS for DN3 comparisons of Foxn $7^{W T / W T}$ versus Foxn 1089/1089; $P=0.002$ for DN4 comparisons of Foxn $1^{933 / 933}$ versus Foxn $1^{933 / 1089}$ and $P=$ NS for DN4 comparisons of Foxn $7^{W T / W T}$ versus Foxn 1089/1089. ND, not detected; PE, phycoerythrin. ferential consequences for early $\mathrm{T}$ cell development and positive selection. We further explored this by characterizing the 4 stages of DN thymocyte progression as DN1-DN4 (DN1: CD44 ${ }^{+} \mathrm{CD} 25^{-}$, DN2: CD 44+CD25+, DN3: CD44-CD25+, DN4: CD44-CD25-). We noted similar percentages of each of the DN1-DN4 subsets when we compared Foxn 1089/1089 thymocytes with those of normal controls (Figure 3B). However, the mouse line that genocopied Pt. 1 (Foxn1 ${ }^{933 / 1089}$ ) had a selective block at the DN1 stage (Figure 3B). The sparse cells in the Foxn $1^{933 / 933}$ line were mainly CD $45^{-}$, revealing a severe lack of immature DN thymocytes. We assessed the effect on positive selection by screening for the upregulation of CD69 on cells expressing intermediate levels of TCR $\beta$ (Figure 3C). The percentage of $\mathrm{CD}^{6} 9^{+}$thymocytes expressing intermediate or high levels of the TCR $\beta$ subunit was reduced in the Foxn 1 
A
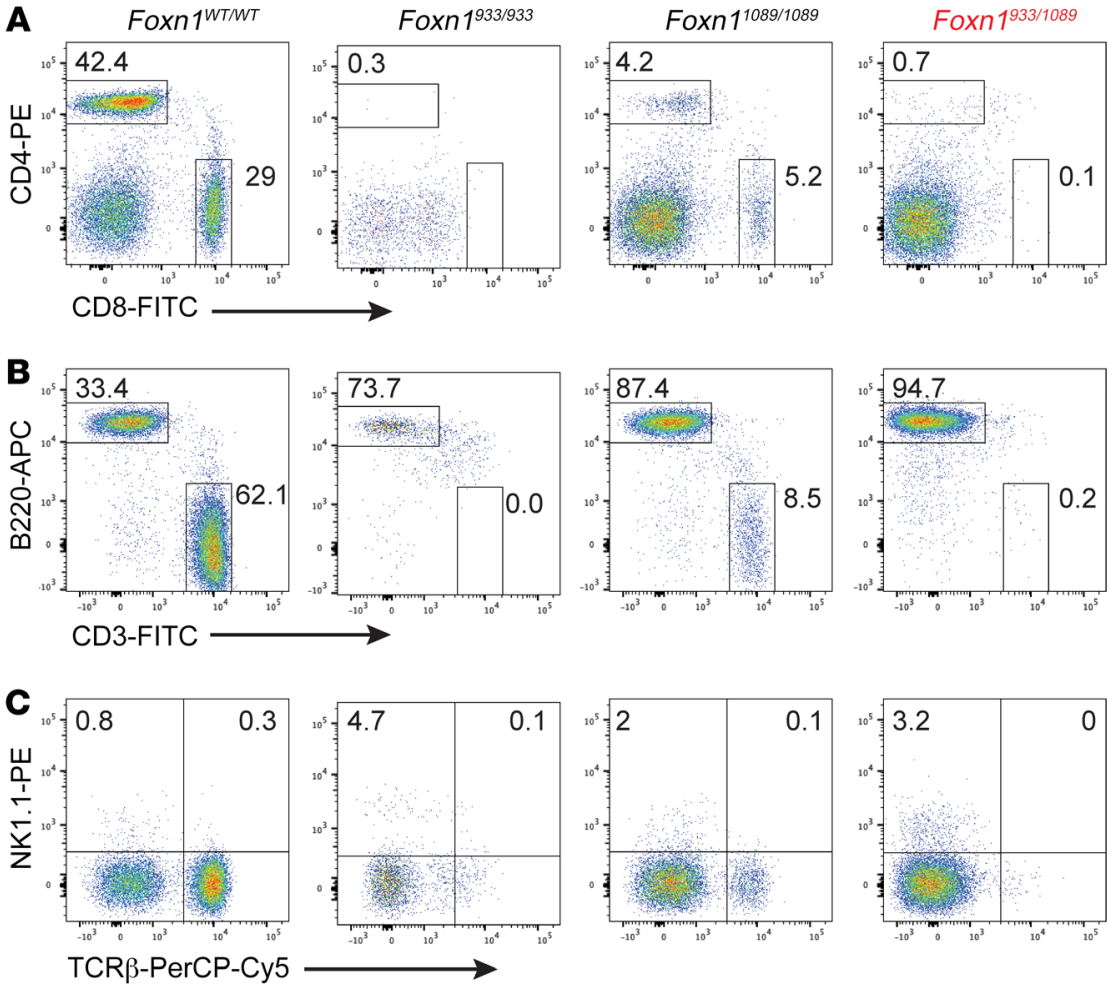

Figure 4. Mice with compound heterozygous mutations in Foxn1 have severe peripheral $T$ cell lymphopenia. (A-C) Lymph nodes were collected from the indicated mice and stained with antibodies detecting cell-surface CD4, CD8, B220, TCR $\beta$, and NK1.1 expression. The cells were analyzed by flow cytometry comparing the cell-surface expression of (A) CD4 ${ }^{+}$and $C D 8^{+}$SP cells; (B) B220 (marker of B cells) and CD3\& cell-surface expression; and (C) NK1.1 cell-surface expression and TCR $\beta . n=3$ to 26 mice per genotype for $\mathbf{A}-\mathbf{C}$. APC, allophycocyanin. (D) The percentages of $\mathrm{CD}^{+}{ }^{+} \mathrm{SP}$ cells, CD8 ${ }^{+} \mathrm{SP}$ cells, B200+ $\mathrm{B}$ cells, and $\mathrm{CD} 3 \varepsilon^{+}$T cells were calculated; $n=24$ Foxn $1^{W T / W T}$, $n=5$ Foxn 1933/933, $n=11$ Foxn 1089/1089, and $n=21$ Foxn $1^{933 / 1089}$ mice. The percentages of NK1.1 $1^{+}$TCR $\beta$ cells were calculated; $n=26$ Foxn $1^{W T / W T}$, $n=3$ Foxn1 ${ }^{933 / 933}, n=11$ Foxn1 1089/1089, and $n=11$ Foxn $1933 / 1089$ mice. For the comparisons shown, a Brown-Forsythe and Welch's 1-way ANOVA was applied. $P$ values of less than 0.05 were considered significant.
D
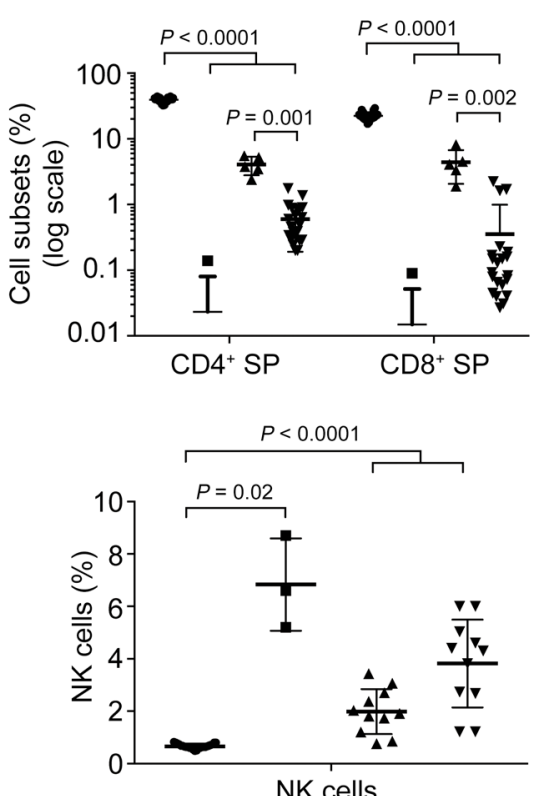

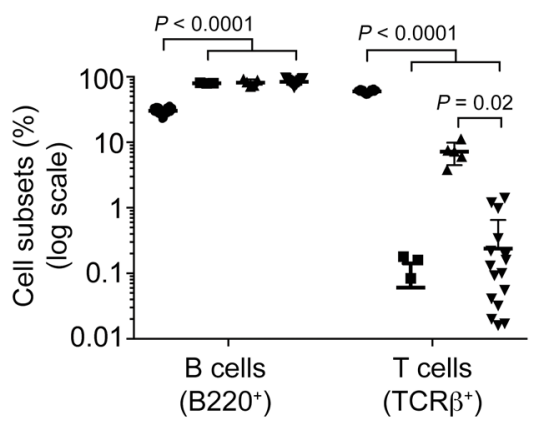

Foxn1WTwt

Foxn $1^{933 / 933}$

Foxn11089/1089

Foxn 1933/1089 heterozygous mouse line $\left(F_{\left.0 x n^{933 / 1089}\right)}\right.$ and in one of the homozygous mutant lines (Foxn1 ${ }^{1089 / 1089}$ ) (Figure 3C). These developmental abnormalities were of statistical significance, as revealed by comparisons of the different thymocyte subpopulations with multiple mice per group (Figure 3D).

The consequence of the thymic aplasia (Foxn $1^{933 / 933}$ mice) and hypoplasia (Foxn $1^{1089 / 1089}$ and Foxn $1^{933 / 1089}$ lines) is an almost complete loss of mature $\mathrm{CD}^{+}$and $\mathrm{CD} 8^{+} \mathrm{SP}$ T cells in the peripheral lymph nodes of these mice (Figure 4A). B cells and NK cells were present, confirming the $\mathrm{T}^{-} \mathrm{B}^{+} \mathrm{NK}^{+}$SCID phenotype of Pt. 1 (Figure
4, B-D). Comparison of the cell populations using 3-26 mice per group confirmed the statistical significance of the findings (Figure 4D). The various cell populations in 6-week-old heterozygous mice (Foxn1 ${ }^{W T / 933}$ and Foxn1 ${ }^{W T / 1089}$ ) were similar to normal control cell populations, revealing that 1 functional allele of Foxn1 was sufficient to support normal T cell development (Supplemental Figure 2, B-E). Taken together, our data demonstrate that $\mathrm{T}$ cell development is severely compromised in mice with compound heterozygous mutations in Foxn1 matching Pt. 1 and results in severe T cell lymphopenia. 
A
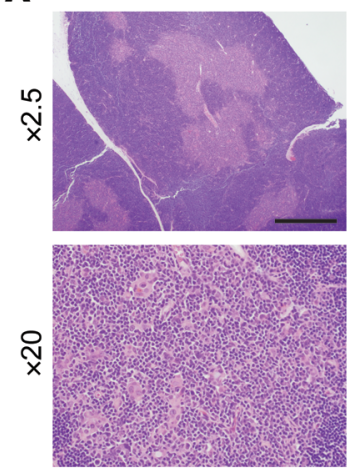

B
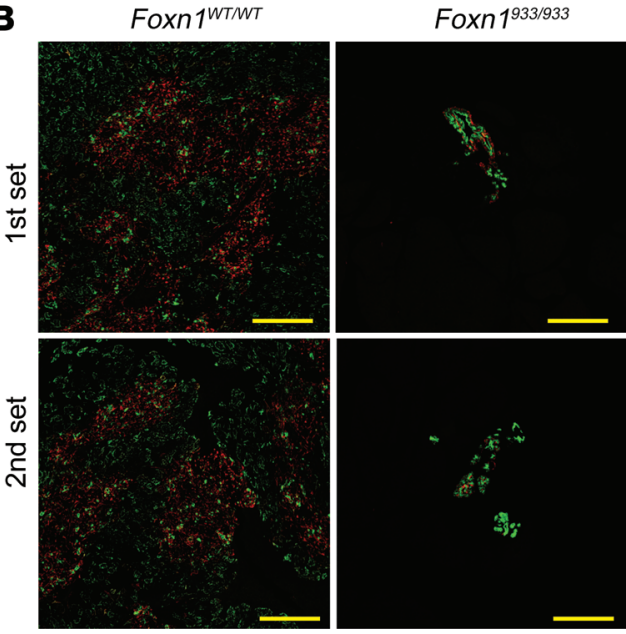

Green $=$ cytokeratin 8 (cTECs);
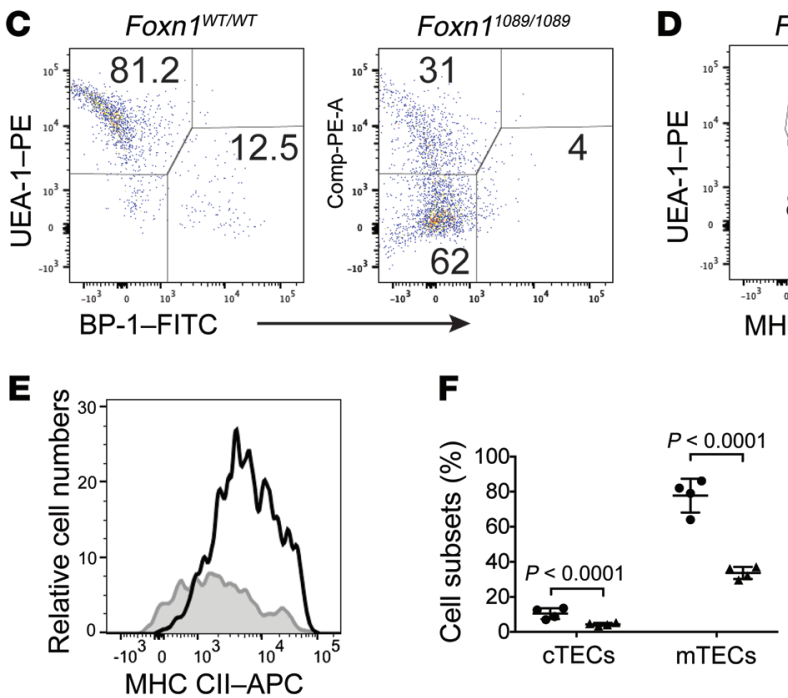

Foxn1 $1089 / 1089$
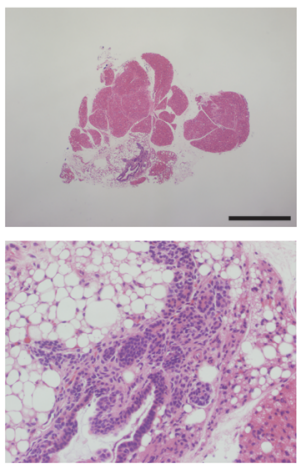

Foxn $1^{933 / 933}$
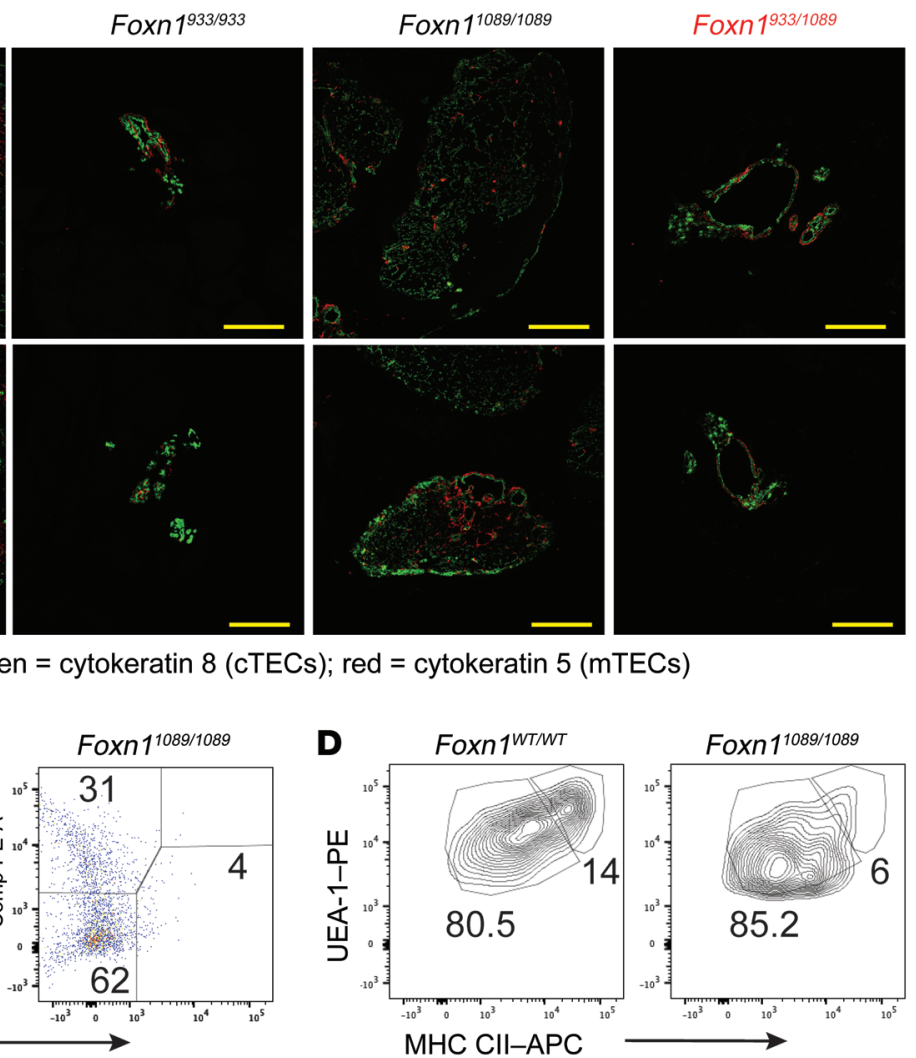

Foxn1933/1089

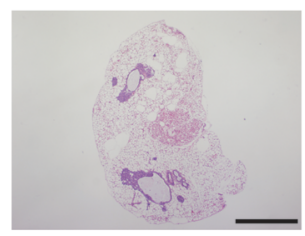

(mTES)

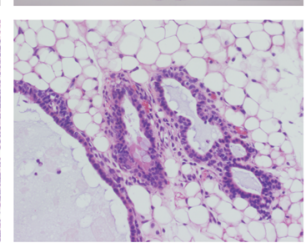

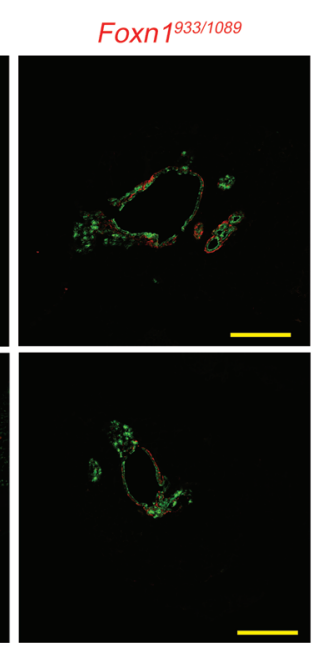
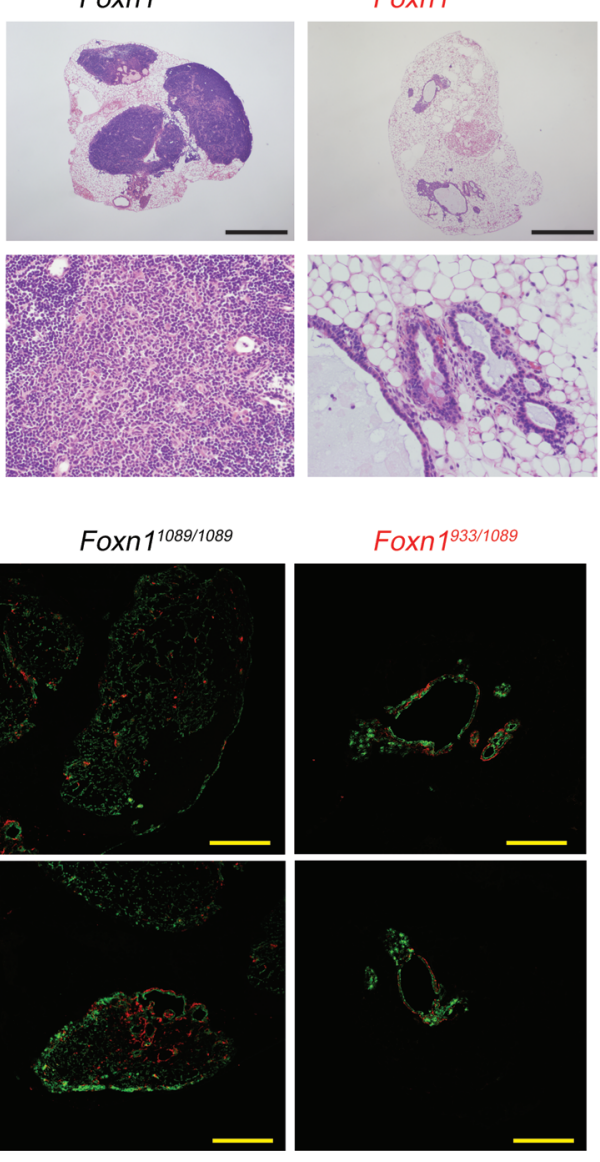
A

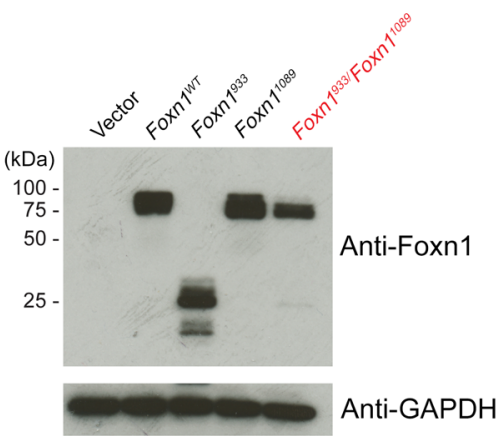

B

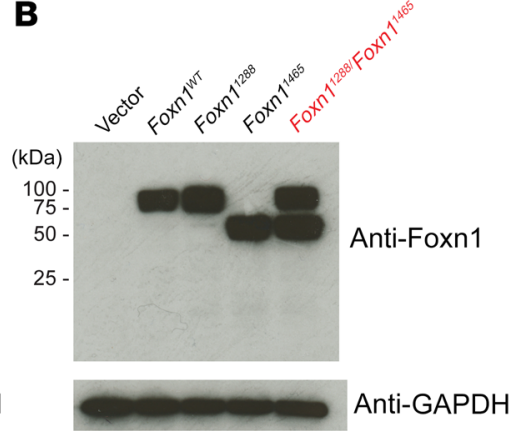

C

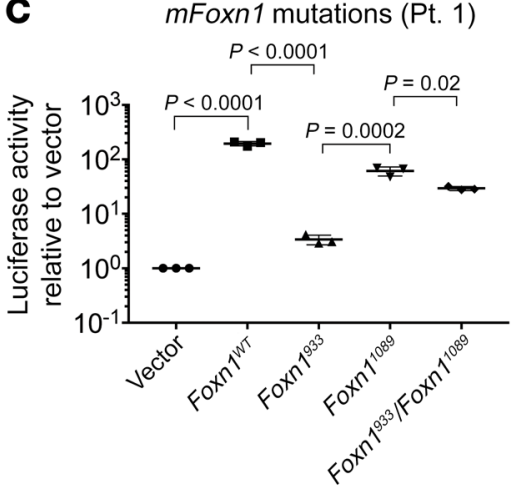

D $\quad$ mFoxn1 mutations (Pt. 2)

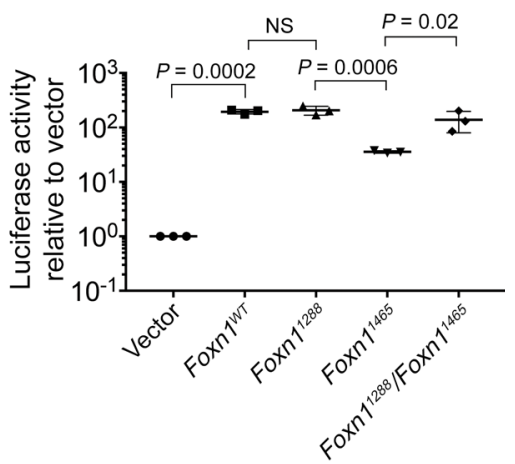

Figure 6. Functional impairment of Foxn1 is dependent on the location and type of mutation. (A-C) Transient transfection assays in HEK293T cells were performed with expression vectors for WT Foxn1 or Foxn1 constructs harboring the indicated mutations that matched the FOXN1 mutations identified in patients. The mutations were divided into those identified in Pt. 1 (A) (Foxn1933, Foxn 1089) and Pt. 2 (B) (Foxn1 1288, Foxn1 1465) as well as the indicated controls. Forty-eight hours after transfection, the cells were lysed, and the proteins were extracted and resolved by SDS-PAGE. Western blotting was performed with antibodies against Foxn1, followed by antibodies detecting GAPDH, which was used as a loading control. Blots are representative of 4 independent experiments. (C and D) HEK293T cells were transfected with the indicated constructs along with a Psmb11 luciferase reporter construct and a $\beta$-gal vector. The mutations (mFoxn1) were grouped for those identified in Pt. 1 (C) (Foxn1 ${ }^{933}$, Foxn 1089) and Pt. 2 (D) (Foxn1 ${ }^{1288}$, Foxn 1465) along with controls (D). Forty-eight hours after transfection, the cells were harvested, and luciferase activity was measured. The luciferase activity was normalized to $\beta$-gal, which was used as an internal control. Data are representative of triplicate samples from each group using 3 independent transfections per group. $P$ values were determined using a standard 1-way ANOVA. approximately $13 \%$ and $81 \%$ cTECs and mTECs (EpCam $\left.{ }^{+} \mathrm{CD} 45^{-}\right)$, respectively (Figure 5, C and F). In contrast, the Foxn $1^{1089 / 1089}$ thymi had $4 \%$ and $31 \%$ cTEC and mTEC representation, respectively (Figure 5F). The lower percentages in the Foxn1 ${ }^{1089 / 1089}$ mice were matched with an increased percentage of EpCam ${ }^{+} \mathrm{CD} 45^{-}$TECs from the Foxn $1^{1089 / 1089}$ mouse line (62\%), which lacked the cytokeratin markers that distinguish cTECs and mTECs (Figure 5C). In normal mice, the mTECs were divided into the less mature MHC class $\mathrm{II}^{\mathrm{lo}}\left(\mathrm{MHC} \mathrm{CII}^{\mathrm{lo}}\right)$ and more mature MHC class $\mathrm{II}^{\mathrm{hi}}\left(\mathrm{MHC} \mathrm{CI}^{\mathrm{hi}}\right)$ cells (Figure 5, D and E). We detected a significant reduction in the percentage of MHC CII ${ }^{\text {hi }}$ cells in the Foxn1 $1^{1089 / 1089}$ lines, and the MHC CII cells present had a reduced cell-surface expression of this molecule relative to littermate controls (Figure 5, D and E). These TEC comparisons indicate that the mutations at the 1089 position of FOXN1 are not as damaging as those that disrupt the DNA-binding domain (position 933), providing some functionality for TEC development.

The transcriptional activity of Foxn1 is regulated by both the DNA-binding and transactivation domains. To determine how the distinct FOXN1 mutations identified in Pt. 1 and Pt. 2 affect the expression and function of the protein, we introduced substitutions or deletions into the cDNA of murine Foxn1 and performed protein expression and promoter-based reporter assay comparisons (2, 39). The insertion of the 4-nt sequence identified in Pt. 1 (Foxn1 ${ }^{933}$ ) resulted in the expression of a truncated $50-\mathrm{kDa}$ protein (Figure 6A). The 15-nt deletion, corresponding to the one identified in the second allele of Pt. 1 (Foxn $1^{1089}$ ), was almost identical in molecular mass to that of the WT control (Figure 6A). Foxn $1^{1288}$ was also similar in size to Foxn $1^{W T}$, whereas a single nucleotide deletion at position 1465 created a truncated protein with a mass of $65 \mathrm{kDa}$ as a result of the frameshift (Figure 6B). As our studies were progress- ing, several additional FOXN1 mutations were identified in unrelated patients presenting with low $\mathrm{T}$ cell counts for whom exome sequencing was undertaken (Table 2 and Supplemental Table 1). Unlike the compound heterozygous genotypes for Pt. 1 and Pt. 2, these patients had FOXN1 mutations evident only on a single allele (Table 2 and Supplemental Table 2). One subject had a previously reported R32OW mutation in FOXN1, whereas 2 patients had the 1465delC that was noted in Pt. 2. We characterized FOXN1 with SNPs (FOXN1 $\left.1^{724}, F O X N 1^{958}, F O X N 1^{962}, F O X N 1^{982}, F O X N 1^{1075}\right)$ and deletions (FOXN1 $\left.1^{12011216}, F O X N 1^{1293 d e l C}, F O X N 1^{1418 d e l C}\right)$, with the human mutations introduced into the murine cDNA (Supplemental Table 2). Consistent with the location and type of mutation, either full-length or truncated Foxn1 proteins were expressed (Supplemental Figure 3, A and B).

The functional activity of Foxn1 mutations corresponding to those in Pt. 1 and Pt. 2, along with the additional mutations, were compared by transcriptional reporter assays using the promoter sequence for the Psmb11 gene, which encodes $\beta 5 t$ (2). We found that Foxn1 ${ }^{933}$ had virtually no transcriptional activity (Figure 6C). The Foxn1 constructs with mutations at positions 1089 and 1465 had $31 \%$ and $18 \%$ WT activity, respectively (Figure 6D). Interestingly, coexpression of the corresponding heterozygous mutation (Foxn $1^{1089}$ with Foxn1 ${ }^{933}$ ) or the polymorphism (Foxn1 ${ }^{1288}$ with Foxn1 ${ }^{1465}$ ) did not attenuate the transcriptional activity of the functional allele, suggesting that Foxn1 functions as a monomer (Figure 6, C and D). Foxn1 $1^{958}$ was inactive, whereas Foxn1 ${ }^{724}$ and Foxn1 $1^{962}$ had activity comparable to that of Foxn1 $1^{W T}$, at $95 \%$ and $82 \%$ normal values, respectively (Table 2 and Supplemental Figure 3C). Foxn1 $1^{982}$ and Foxn1 $1^{1075}$ exhibited $16 \%$ and $63 \%$ control activity, respectively (Table 2 and Supplemental Figure 3C). The deletions at positions 1201, 1293, and 1418 had a more severe effect, 
A

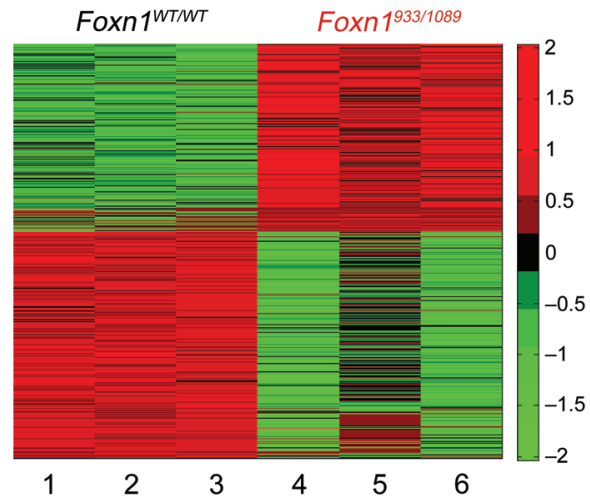

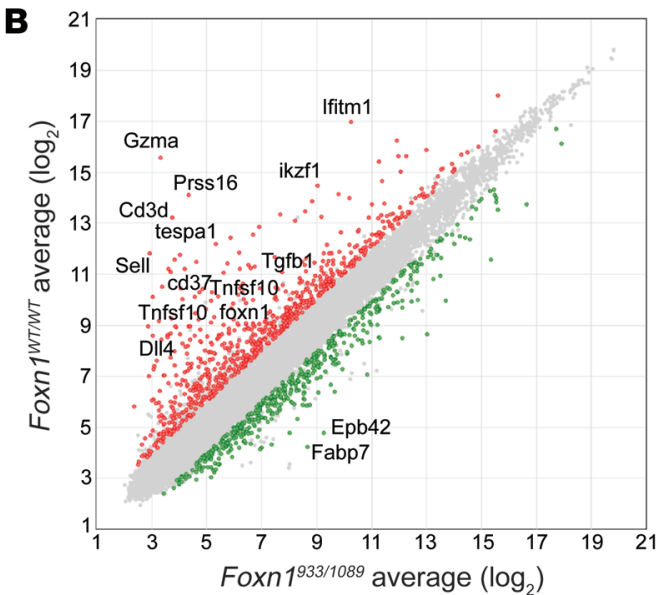

C P4 hair extrusion
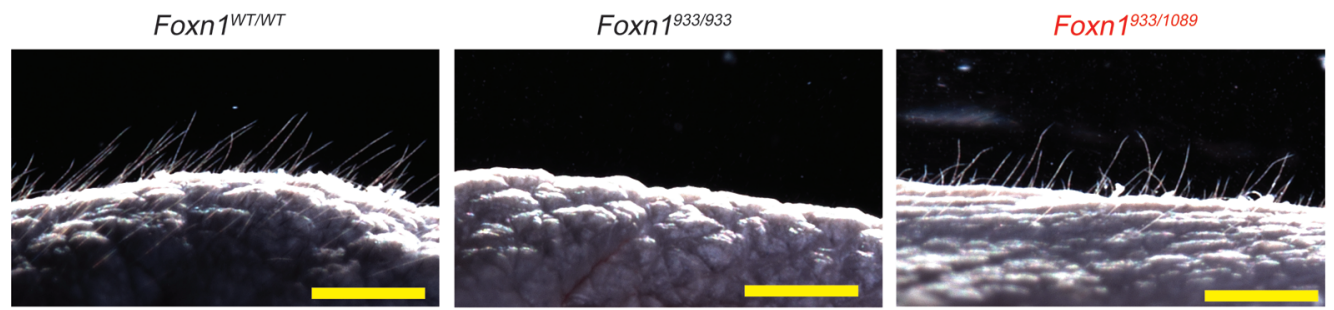

D Skin: control vs. nude/SCID

E Skin: control vs. Pt. 1 genocopy
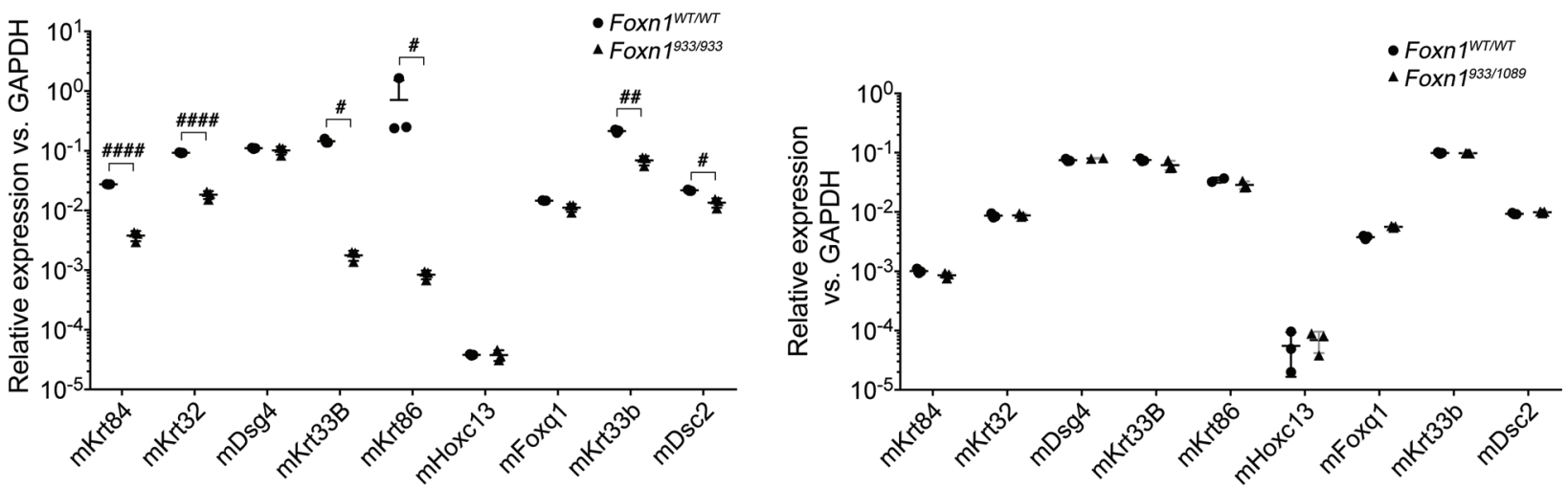

Figure 7. Differential functions of Foxn1 in TECs versus keratinocytes. (A) Gene expression comparisons were made with 3 independently isolated fetal thymi, obtained from E13.5 embryos of the indicated genotypes. The presence of a hypoplastic thymi was confirmed by subsequent genotyping to select for Foxn $1^{933}$ and Foxn $1^{1089}$ alleles. A heatmap revealed a subset of up- and downregulated genes, with a selection criterion of a 1.5 -fold difference as a cutoff. (B) Data on differentially expressed transcripts, particularly those with reported functions in embryonic thymic development. (C) Images of postembryonic day-4 mice of the indicated genotypes were processed with bright-field imaging on a dark background, revealing hair and fur extending out from the skin. Scale bars: $1 \mathrm{~mm}$. (D and E) qRT-PCR was performed to compare the levels of genes previously reported to be involved in hair shaft extension. This was performed to compare (D) 3 littermate controls and 3 Foxn $1^{933 / 933}$ mice and, in a separate experiment, (E) 3 controls and 3 Foxn $1^{933 / 1089}$ mice. The data

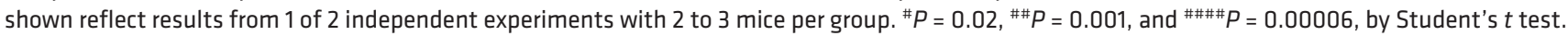
There were no significant differences in any of the transcripts compared between the 2 mouse lines in $\mathbf{E}$.

reducing the functional activities of Foxn1 to $3 \%, 2 \%$, and $12 \%$ of normal values, respectively (Table 2 and Supplemental Figure 3D). Taken together, these results confirm crucial roles for both the DNA-binding and transactivation domains in the transcriptional activity of Foxn1, with the extent of functional incapacitation being dependent on the location and type of Foxn1 mutation (Figure 1C and Table 2).

FOXN1 differentially regulates genes in the thymus versus the skin. The Foxn1 ${ }^{933 / 933}$ homozygous knockin mice have a phenotype identical to that of the classic $n u / n u$ mouse, consistent with an essential role for the FOXN1 DNA-binding domain in both TEC and keratinocyte differentiation and gene expression. Contrasting with this, the Foxn1 $1^{933 / 1089}$-knockin mouse line, genocopying Pt. 1, had a normal hair coat and whiskers despite the dramatic thymic hypoplasia. This suggests that the Foxn $1^{933 / 1089}$ mutations transcriptionally affect TECs differently than they do keratinocytes. We considered 2 explanations for the divergent consequences of harboring Foxn1 $1^{933 / 933}$ versus Foxn1 $1^{933 / 1089}$ genotypes for hair and nail 
phenotypes. First, the FOXN1 $1^{933 / 1089}$ mutations could selectively affect the TEC transcriptome as opposed to the keratinocyte transcriptome. Alternatively, the loss-of-function mutations in FOXN1 could modulate RNA transcripts in both TECs and keratinocytes in a quantitative manner, with the latter cell population maintaining reduced yet sufficient levels of transcripts to enable extrusion of the hair shaft and nail beds. To address these possibilities and characterize the transcript changes that resulted from the compound heterozygous mutations in FOXN1, we performed comparative gene expression analyses. RNA was extracted from embryonic thymic lobes at E13.5 and used for gene analyses. The E13.5 time point coincides with an expansion/differentiation phase of TECs prior to significant thymocyte expansion. We made gene expression comparisons with 3 control thymi $\left(F o x n 1^{\text {WT/WT }}\right)$ and 3 hypoplastic thymi (Foxn1 $1^{933 / 1089}$ ) (Figure 7A). Gene array comparisons revealed 417 upregulated and 500 downregulated genes in the Foxn1 $1^{933 / 1089}$ line relative to the littermate controls (Figure 7A). These differences were not surprising, given the severe hypoplasia evident in the Foxn1 $1^{933 / 1089}$ mice (Figure 3B). Our focus on a select subset of transcripts with roles in the development of the thymus and parathyroids, which are linked during the patterning of the third pharyngeal apparatus, revealed some surprising differences (Figure 7B). Tbx1 and Eya1 levels were higher in mutant mice, suggesting impaired differentiation of the thymus within the third pharyngeal pouch region (Figure 7B and Supplemental Table 3). In contrast to this, we found that Pax1 and Dcs2 levels were substantially lower in mutant mice (Supplemental Table 3). Although Pax1 participates in the formation of the thymic lobes, Dsc2 has only been described as a Foxn1 target in the skin (3, 40). Taken together, our data are consistent with previous reports comparing normal and Foxn1-mutant mice and provide further insights into the transcript changes in embryonic hypoplastic thymi (2).

To determine how the same mutations in Foxn1 affect hair follicle development, we isolated skin from 4-day-old pups. This is a stage when hair follicle extrusion and whisker formation is first evident in normal mice (Figure 7C). As previously reported, the Foxn1 $1^{933 / 933}$ pups showed no hair follicle extrusion (Figure 7C). In contrast, the hair in the Foxn1 $1^{933 / 1089}$ mice was visually similar to that of littermate controls (Figure 7C). We performed quantitative reverse transcription PCR (qRT-PCR) to compare a number of key genes expressed along the developing hair shaft needed for hair follicle extrusion. The nude mice (Foxn1 $1^{933 / 933}$ ) had reduced expression of mouse Krt84 (mKrt84), mKrt33, mKrt33b, and mDsc2, consistent with the central role of the DNA-binding activity of Foxn1 in enabling keratinocyte functions (Figure 7D). Foxn1 $1^{933 / 1089}$ mice have no nude phenotype. The transcripts coupled to hair follicle extrusion in these mice were expressed at levels comparable to those seen in littermate controls (Figure 7E). This indicates a functional dichotomy in the requirements for Foxn1 in controlling extrusion of the hair shaft in comparison with expression of thymus-selective genes.

\section{Discussion}

FOXN1 is a master transcriptional regulator of TECs that controls the expression of genes critical for both cTEC and mTEC differentiation and expansion $(1,28,41)$. Although autosomal-recessive mutations in FOXN1 can result in a nude/SCID phenotype, even hypomorphic alleles can reduce the effectiveness of thymopoiesis
$(2,42)$. This is best revealed in mouse models, in which enforced expression of Foxn1 in aged mice prevents the characteristic age-related thymic involution and improves T cell output (43-45). We identified what to our knowledge are several previously unreported mutations in human FOXN1 and, for 2 patients, distinct compound heterozygous mutations. Genocopying the compound heterozygous mutations identified in Pt. 1 using a mouse model was consistent with a novel clinical presentation of $\mathrm{T}^{-} \mathrm{B}^{+} \mathrm{NK}^{+} \mathrm{SCID}$ without evidence of alopecia or nail dystrophy. We identified the FOXN1 variants in Pt. 2 using targeted exome sequencing. However, this approach did not establish whether both variants were restricted to 1 allele, with the possibility that a normal FOXN1 sequence was present on the second allele. To answer this, we cloned the region comprising exon 8 and used more than 16 independently isolated bacterial clones to sequence the FOXN1 region comprising positions 1288 and 1465 . Consistent with a compound heterozygous genotype, we confirmed individual allelic variants for positions 1288 and 1465 . We also had an unexpected finding of some clones with a WT FOXN1 sequence. Although molecular analysis for maternal engraftment when the child was 2 months of age was negative, we suspect that the WT FOXN1 sequences were maternally derived, since DNA was obtained from the child's blood at 5 months of age (subsequent studies for maternal engraftment were not obtained at this time point). Moreover, the mother did not have any clinical indications of a $\mathrm{T}^{-/ / \mathrm{lo}}$ phenotype. The allelic variant at position 1288 did not affect the expression or function of full-length FOXN1, as established through Western blot analysis and a $\beta 5 t$ luciferase reporter assay. The 1288 polymorphism is in fact reported in approximately $3 \%$ of the general population (dbSNP: rs61749867), suggesting that it is benign. However, the Pro-to-Ser amino acid change due to the 1288 polymorphism would result in a conformational modification in the protein. This change could affect FOXN1 interactions with other proteins involved in thymopoiesis. Consequently, we cannot rule out the possibility that the 1288 variant has a functional impact in vivo in the context of a second allelic FOXN1 pathogenic variant. We are currently testing this in additional mouse models.

Our characterization of the human FOXN1 mutations in mouse models has uncovered what we believe to be a novel region at the $\mathrm{COOH}$-terminal end of the DNA-binding domain that uncouples TEC development from keratinocyte differentiation. Comparison of the thymic hypoplasia in the Foxn1 ${ }^{933 / 1089}$ and Foxn1 $1^{1089 / 1089}$ mouse lines revealed a critical 5-amino acid stretch in Foxn1 that was deleted as a result of the 1089 mutation in Pt. 1. This 5-amino acid segment is highly conserved with Foxn4, an ancestral gene present in all species that evolved with a thymus or thymus-like structure (thymoid) (Supplemental Figure 4) (16). The thymus/thymoid-like structures are present in cartilaginous fish (sharks) and agnathans (lampreys), but not in cephalochordates (segmented marine animals such as lancelets) (19). Interestingly, the 5-amino acid sequence in the lancelet Foxn 4 diverges from that in lamprey, potentially explaining the emergence of the thymus-like structure (16). Possible interactions between this 5-amino acid stretch and distinct transcription factors and/or other DNA-binding proteins could account for the differential function in TECs versus keratinocytes. Although one nuclear localization site (NLS) prediction program suggested that this site was an NLS, we noted that the 
Foxn $1^{1089}$ protein could translocate into the nucleus. Further supporting our suggestion that the nuclear localization remains intact with the 15-nt deletion in Foxn $1^{1089}$ is the observation that the compound heterozygous mice (Foxn1 $1^{933 / 1089}$ ) genocopying Pt. 1 had normal transcript levels of genes necessary for hair follicle extrusion. In most studies to date, Foxn1 appears to have overlapping requirements for both TEC and keratinocyte development (46). To our knowledge, only 1 report has revealed a differential contribution of Foxn1 in TECs versus keratinocytes $(18,47)$. In the published study, the removal of exon 3 selectively caused thymic aplasia, with no effects on hair shaft or nail bed development (47). It remains unknown how the exon 3 deletion results in the differential effects of Foxn1 on TECs versus keratinocytes.

A second important region in Foxn1 is the transactivation domain, encoded within exons 8-9 (18). The compound heterozygous mutations in Pt. 1 are proximal to this transactivation domain. In addition, Pt. 2 presented with a mutation at 1465 , which affects the transactivation domain. The introduction of this 1465 deletion mutation in murine Foxn1 resulted in the expression of a truncated protein that had only $18 \%$ normal transcriptional activity, even though the DNA-binding domain remained intact. This confirms a critical role for the transactivation domain in TEC development, possibly via interactions with a yet-to-be identified coactivator. Alternatively, the mutations near the transactivation domain may provide additional DNA-binding specificity for induction of TEC-selective genes, and keratinocytes may not require this binding specificity.

Several additional patients were identified through SCID screening of newborns with low TREC numbers and were found to have mutations on just 1 allele of FOXN1. These mutations affected multiple regions in FOXN1, including the DNA-binding and/or transactivation domains (Table 2 and Supplemental Table 2). Although not predicted to be causal to a SCID phenotype because of the presence of $1 \mathrm{WT}$ allele, such mutations could lead to reduced $\mathrm{T}$ cell output, either during stress at birth or through aging. In mouse models, hypomorphic mutations in murine Foxn1 result in thymic involution as the mice age, contributing to reduced T cell output $(42,45,48,49)$. It remains to be seen whether patients with single-allele mutations in FOXN1 have an earlier onset of thymic involution at birth due to stress and later in life. Although our comparisons of 24-week-old Foxn1 heterozygous mice $\left(F o x n 1^{933 / W T}\right)$ failed to reveal evidence of premature thymic involution, the impact of stress on thymopoiesis still needs to be assessed in these mice (50). Among the patients with single-allele mutations were 2 individuals with the same mutation as that in Pt. 2, at position 1465. Pt. 7 has a mutation in the DNA-binding region of FOXN1 and was clinically reported to have very low $\mathrm{T}$ cell numbers and normal hair and nail beds. Such findings suggest that additional polymorphisms and/or mutations in noncoding regions of FOXN1 (intron, promoter, enhancer elements) could contribute to the low $\mathrm{T}$ cell counts noted in those with only 1 affected allele. Alternatively, mutations in other genes coupled to FOXN1 functions may further compound the effects of the identified singleallele mutations, as exemplified with the reported DOCK8 and/or CHD7 mutations in 2 of the patients (Table 2).

In summary, we describe what we believe to be a new clinical presentation of a primary immunodeficiency related to com- pound heterozygous mutations in FOXN1, causing $\mathrm{T}^{-/ 10} \mathrm{~B}^{+} \mathrm{NK}^{+}$ SCID without the typical nude phenotype. Therefore, a careful structure-function analysis is warranted before assuming that low TREC and T cell counts in patients are caused by FOXN1 mutations, particularly in individuals with monoallelic mutations. Consideration of maternal $\mathrm{T}$ cell engraftment is also important if most of the cells are memory T cells and a low TREC output is maintained. A challenge is to know whether a given FOXN1 mutation may allow for the development of sufficient numbers of $\mathrm{T}$ cells to prevent infections. Thymus transplantation should not be undertaken if the $\mathrm{T}$ cell numbers are likely to increase with time (51, 52). In view of the likelihood that the 2 alleles of FOXN1 produce proteins that function independently, patients with single-allele mutations should be followed at least for several months to a year prior to considering thymus transplantation.

\section{Methods}

Antibodies, oligonucleotides, and plasmids. The antibodies used in the experiments as well as their sources and the plasmids used for transfections are listed in Supplemental Table 4. Oligonucleotide sequences used for genotyping, PCR reactions, qRT-PCR reactions, and sequencing are listed in Supplemental Table 5. The murine Foxn1 cDNA was cloned from genomic DNA using E14.5 fetal thymi isolated from C57BL/6 timed pregnant mice. The sequence was confirmed by Sanger sequencing after the RT-PCR product was subcloned into the pCR2.1-TOPO TA cloning vector (Thermo Fisher Scientific). The murine Foxn1 cDNA was subcloned into the pCMV-FLAG-vector (MilliporeSigma) using the Bgl II/Kpn I restriction enzyme cloning sites. Site-directed mutagenesis was performed using the QuikChange Site-Directed Mutagenesis Kit (Stratagene). The human FOXN1 mutations were introduced into the murine equivalent location with complementary oligonucleotide primers, which are listed in Supplemental Table 5. All mutated sequences were confirmed with DNA sequencing. Exon 8 from genomic DNA obtained from Pt. 2 was prepared and subcloned by PCR reactions using LA-Taq (Takara). The PCR reactions were resolved on agarose gels, and a 479bp fragment was isolated and subcloned into pCR2.1 using the TOPOTA Cloning System (Thermo Fisher Scientific). Eighteen independent clones were expanded and used for sequencing with $\mathrm{T} 7$ primers.

Thymocyte and EC preparations. Thymocyte and peripheral $\mathrm{T}$ cell populations were processed and stained for flow cytometric analyses as previously described $(53,54)$. TECs were isolated from individual thymic lobes by digestion in Liberase (Roche) in the presence of DNase I (Roche) as described previously (55). The cells were stained with antibodies against CD45 (Tonbo Scientific), MHC CII (I-A/I-E) (Tonbo Scientific), EpCAM (eBioscience), BP-1 (eBioscience), and UEA-1 (Vector Laboratories). Samples were analyzed on a FACSCanto II Flow Cytometer (BD Bioscience. FlowJo software (Tree Star) was used to analyze the flow data. TEC subsets were analyzed by selection of $\mathrm{CD}^{-} 5^{-} \mathrm{EpCAM}^{+}$ $\mathrm{MHCII}^{+}$cells with either $\mathrm{UEA} 1^{+} \mathrm{BP}^{-}$for medullary $\mathrm{TECs}$ or $\mathrm{BP}^{+} \mathrm{UEA1}^{-}$ for cortical TECs. MHC CII ${ }^{\text {hi }}$ and $\mathrm{MHC} \mathrm{CII}{ }^{\text {lo }}$ cells were used to discriminate between the 2 cortical and 2 medullary TEC subsets. Thymocyte subsets and peripheral $\mathrm{T}$ cells were analyzed for cell-surface expression of various proteins including CD3, CD4, CD8, CD11b, CD11c, CD19, CD25, CD44, CD45, CD69, B220, NK1.1, $\alpha \beta$ TCR, $\gamma \delta$ TCR, and Ter119 (see Supplemental Table 4 for antibody sources).

RNA isolation and analysis. RNA was isolated from adult thymic tissues and P4 skin with miRNAeasy kits (QIAGEN). The tissues were 
initially homogenized with a Dounce homogenizer in the presence of QIAzol (QIAGEN). For embryonic thymi, a miRVANA RNA Isolation Kit (Ambion, Thermo Fisher Scientific) was used to isolate the RNA. Contaminating DNA from all the RNA preparations was eliminated by DNAse I digestion (Ambion, Thermo Fisher Scientific) using incolumn digestion and/or DNAse treatment followed by purification with an RNA clean-up step (RNA-5 Quick and Clean, Zymo Research Inc.). cDNA was generated with 100 ng to $1 \mu$ g RNA using the High-Capacity cDNA Reverse Transcription Kit (Applied Biosystems). Random priming was used for reverse transcription. qRT-PCR was performed with $100 \mathrm{ng}$ cDNA using SYBR Green (Thermo Fisher Scientific). The various PCR reactions and conditions were performed as described previously (56). For gene expression comparisons, the Affymetrix Murine Clariom S mouse array was used, which focuses on 20,000 well annotated genes (Thermo Fisher Scientific). The data on comparisons of the differentially expressed transcripts are available in the Gene Expression Ontology (GES) database (GES 134458; https:// bioportal.bioontology.org/ontologies/GEXO).

IHC and Western blot analysis. Thymic tissue was fixed and embedded in paraffin. H\&E staining was performed as standard protocol, and tissue was imaged on an Axiovert 200M inverted fluorescence microscope. For immunofluorescence staining, sections were incubated with rabbit anti-cytokeratin 5 (1:100, Abcam) and mouse anti-cytokeratin 8 (1:100, BioLegend) at $4^{\circ} \mathrm{C}$ overnight. The next day, sections were washed with PBS and stained with goat anti-mouse AF488 and donkey anti-rabbit AF555 secondary antibody (1:200, Invitrogen, Thermo Fisher Scientific). Tissues were then stained with DAPI and mounted with ProLong Gold Antifade Mountant with DAPI (Thermo Fisher Scientific). Images were taken on a Leica confocal microscope. Western blotting was performed as described elsewhere using RIPA lysis procedures with antibodies against Foxn1, GAPDH, and the FLAG epitope listed in Supplemental Table 4 (57).

Transfections and luciferase reporter assays. A luciferase reporter containing the promoter of the Psmb11 gene was provided by S. Zuklys and G. Holländer (University of Oxford, Oxford, United Kingdom) (2). The Psmb11 luciferase reporter construct $(0.5 \mu \mathrm{g})$ was cotransfected into HEK $293 \mathrm{~T}$ cells $\left(2.5 \times 10^{5}\right.$ cells/well $)$ together with pCMV-FLAG $(0.5 \mu \mathrm{g})$ expression vectors containing Foxn1 WT or mutants, using Fugene 6 Reagent (Promega). A separate construct containing $\beta$-gal $(0.1 \mu \mathrm{g})$ was included in the transfections to normalize each well for transfection efficiency. Forty-eight hours after transfection, the cells were harvested and the luciferase activity measured using a luciferase assay kit (Promega). Luciferase activity was normalized to $\beta$-gal activity, which was used as an internal control.

Statistics. Statistical analyses were performed using GraphPad Prism software, versions 7.0d and 8.2 (GraphPad Software). A $P$ value of less than 0.05 was considered statistically significant. For Figure 2D, Figure 3D, and Figure 4D, a Brown-Forsythe and Welch's 1-way ANOVA was applied instead of an ordinary 1-way ANOVA, since the variances were quite extreme, because the various knockin mouse groups had extremely low cell numbers and percentages relative to the control Foxn1 $1^{\text {WT/WT }}$ line (ordinary ANOVA assumes equal variances). For Figure 6, C and D, a normal 1-way ANOVA analysis was applied. Standard Student's $t$ tests were used for data analysis in Figure 5F and Figure 7, D and E.

Study approval. The IRB of UT Southwestern Medical Center approved this study (approval nos. 072010-009 and 112010-013). MLM, GTP, and TRY acquired a limited set of data on different
FOXN1 patients in the United States. Consent for sequencing of DNA from Pt. 2 was obtained. Sequence information on deidentified Pts. 3-16 were obtained under a protocol declared exempt by the IRB of the Duke University Health System. The data were forwarded by various clinicians throughout the United States. Animal experiments described in this work were approved by and conducted with oversight of the IACUC of UT Southwestern (APN nos. 2015-101247 and 2015-101163). Mice were housed in a specific pathogen-free facility at UT Southwestern Medical Center. The Foxn1-targeted mice were developed entirely on a C57BL/6 background. Although more than 40 founders were screened for each construct, 2 independent mouse lines were expanded for each of the mutations generated by CRISPRCas: the Foxn1 $1^{933}$ mutations (Foxn1-933 no. 17 and Foxn1-933 no. 30) and the Foxn1 ${ }^{1089}$ mutations (Foxn1-1089 no. B4 and Foxn1-1089 no. B15). The mice are described further in Supplemental Table 4. The Foxn1 mouse lines were crossed for 1 to 2 generations with C57BL/6 mice prior to intercrossing.

\section{Author contributions}

MTDLM and CMS provided care for Pt. 1 and Pt. 2, respectively. MLM, GTP, and TRY acquired deidentified genetic data under an IRB-exempt protocol for Pt. 3-Pt. 9, who were found to have mutations in FOXN1. QD, LKH, MLM, MDLM, and NSCVO conceived the experiments. QD, LKH, FC, ID, PR, EM, MAK, and NSCVO performed the experiments. ID, PR, and SK analyzed the RNA-Seq and array data. QD, LKH, FC, PR, ID, GTP, TRY, MLM, MTDLM, CAW, and NSCVO analyzed the data. QD, MDLM, and NSCVO wrote the manuscript. MLM and CMS reviewed the manuscript.

\section{Acknowledgments}

We would like to thank Angela Mobley and Kayla Lopez from the flow cytometry core at UT Southwestern Medical Center for assistance with TEC sorting and flow analyses. We appreciate the suggestions and help provided by members of the van Oers laboratory. We are thankful for the statistical analysis that was suggested and/or provided by Lindsay Cowell (Department of Bioinformatics, UT Southwestern Medical Center). Caitlyn Braisch from Ondine Cleaver's laboratory at UT Southwestern Medical Center provided image acquisition assistance. We are grateful for the advice provided by Dong-Ming Su (University of North Texas Health Sciences Center) and Nancy Manley (Department of Genetics, University of Georgia). James Richardson, John Shelton, and staff from the Cardiology Core of UT Southwestern Medical Center provided help with thymic tissue and skin processing. The various Foxn1-targeted mice were generated with the help of Robert Hammer of the Transgenic and Knockout Core at UT Southwestern Medical Center. Salius Züklys and Georg Hollander (University Children's Hospital and University of Basel, Basel, Switzerland) provided the $\beta 5 \mathrm{t}$ transcriptional reporter construct. We further acknowledge the following physicians who provided genetic and clinical data on deidentified individuals with diverse FOXN1 mutations. Patient information was provided to M. Louise Markert at Duke University (Durham, North Carolina, USA). The physicians included Jennifer Heimall (Children's Hospital of Philadelphia, Philadelphia, Pennsylvania, USA); Elena Perez (Allergy Associates of the Palm Beaches, North Palm Beach Florida, USA); John Bohnsack and Karin Chen (University of Utah Health, Salt 
Lake City, Utah, USA); Challice Bonifant (C.S. Mott Children's Hospital, Ann Arbor, Michigan, USA); Hana Niebur (Children's Hospital and Medical Center, Omaha, Nebraska, USA); Jordan Abbott and Erwin W. Gelfand (National Jewish Health, Denver, Colorado, USA); Melissa E. Elder (University of Florida, Gainesville, Florida, USA); Lisa Forbes and Jacob J.H. Bleesing (University of Cincinnati, Cincinnati, Ohio, USA); David Buchbinder (Children's Hospital of Orange County, Orange, California, USA); Melanie Hankewzcz (Hackensack Meridian, Hackensack, New Jersey, USA); Chaim Roifman (Sick Kids, Toronto, Canada); Evan B. Shereck (Oregon Health and Science University, Portland, Oregon, USA); Megan Cooper (St. Louis Children's Hospital, St. Louis, Missouri, USA); Lisa Bartnikas (Boston Children's Hospital, Boston, Massachusetts, USA); Rebecca H. Buckley (Duke University Medical Center, Durham,North Carolina, USA); Jennifer Leiding (University of South Florida, Saint Petersburg, Florida, USA); Anthony Hayward (Brown University, Providence, Rhode Island, USA); Benjamin T. Prince (Nationwide Children's Hospi- tal, Columbus, Ohio, USA); Neena Kapoor (Children's Hospital of Los Angeles, Los Angeles, California, USA); Mark T. Vander Lugt (C.S. Mott Children's Hospital, Ann Arbor, Michigan, USA); and Tory Quigg (Methodist Children's Hospital, San Antonio, Texas, USA). Our work was supported in part by grants from the NIH (R01 AI114523 and R21 AI144140, to NSCVO); Beecherl funds from the Department of Immunology at UT Southwestern Medical Center (to NSCVO); and the Jeffrey Modell Foundation (to MDLM and CAW). Our work was also supported, in part, by grants from the NIH R01 (R01 AI114523, R21 AI144140 NSCVO), and the Jeffrey Modell Foundation (MDLM).

Address correspondence to: Nicolai S.C. van Oers, NA2.200, 6000 Harry Hines Boulevard, Dallas, Texas, 75390-9093, USA. Phone: 214.648.1236; Email: Nicolai.vanoers@utsouthwestern.edu.

QD's present address is: Harbour Biomed, Newton, Massachusetts, USA.
1. Vaidya HJ, Briones Leon A, Blackburn CC. FOXN1 in thymus organogenesis and development. Eur JImmunol. 2016;46(8):1826-1837.

2. Žuklys S, et al. Foxn1 regulates key target genes essential for $\mathrm{T}$ cell development in postnatal thymic epithelial cells. Nat Immunol. 2016;17(10):1206-1215.

3. Gordon J, Manley NR. Mechanisms of thymus organogenesis and morphogenesis. Development. 2011;138(18):3865-3878.

4. Anderson G, Takahama Y. Thymic epithelial cells: working class heroes for T cell development and repertoire selection. Trends Immunol. 2012;33(6):256-263.

5. van der Spek J, Groenwold RH, van der Burg M, van Montfrans JM. TREC based newborn screening for severe combined immunodeficiency disease: a systematic review. JClin Immunol. 2015;35(4):416-430.

6. Abramson J, Anderson G. Thymic epithelial cells. Annu Rev Immunol. 2017;35:85-118.

7. Satoh R, et al. Requirement of Stat3 signaling in the postnatal development of thymic medullary epithelial cells. PLoS Genet. 2016;12(1):e1005776.

8. Anderson MS, Venanzi ES, Chen Z, Berzins SP, Benoist C, Mathis D. The cellular mechanism of Aire control of $\mathrm{T}$ cell tolerance. Immunity. 2005;23(2):227-239.

9. Takaba H, et al. Fezf2 orchestrates a thymic program of self-antigen expression for immune tolerance. Cell. 2015;163(4):975-987.

10. Aschenbrenner K, et al. Selection of Foxp $3^{+}$regulatory $\mathrm{T}$ cells specific for self antigen expressed and presented by Aire+ medullary thymic epithelial cells. Nat Immunol. 2007;8(4):351-358

11. Liu Z, Gerner MY, Van Panhuys N, Levine AG, Rudensky AY, Germain RN. Immune homeostasis enforced by co-localized effector and regulatory T cells. Nature. 2015;528(7581):225-230.

12. Nehls M, Pfeifer D, Schorpp M, Hedrich H, Boehm T. New member of the winged-helix protein family disrupted in mouse and rat nude mutations. Nature. 1994;372(6501):103-107.

13. Hannenhalli S, Kaestner KH. The evolution of
Fox genes and their role in development and disease. Nat Rev Genet. 2009;10(4):233-240.

14. Gajiwala KS, et al. Structure of the winged-helix protein hRFX1 reveals a new mode of DNA binding. Nature. 2000;403(6772):916-921.

15. Bajoghli B, et al. A thymus candidate in lampreys. Nature. 2011;470(7332):90-94.

16. Swann JB, et al. Conversion of the thymus into a bipotent lymphoid organ by replacement of FOXN1 with its paralog, FOXN4. Cell Rep. 2014;8(4):1184-1197.

17. Newman JA, et al. The structural basis for forkhead box family specificity revealed by the crystal structure of human FOXN1 in complex with DNA. bioRxiv. https://doi.org/10.1101/428011. Accessed September 26, 2018. Accessed August 22, 2019.

18. Schüddekopf K, Schorpp M, Boehm T. The whn transcription factor encoded by the nude locus contains an evolutionarily conserved and functionally indispensable activation domain. Proc Natl Acad Sci U S A. 1996;93(18):9661-9664.

19. Schlake T, Schorpp M, Nehls M, Boehm T. The nude gene encodes a sequence-specific DNA binding protein with homologs in organisms that lack an anticipatory immune system. Proc Natl Acad Sci U S A. 1997;94(8):3842-3847.

20. Flanagan SP. 'Nude', a new hairless gene with pleiotropic effects in the mouse. Genet Res. 1966;8(3):295-309.

21. Wortis HH, Nehlsen S, Owen JJ. Abnormal development of the thymus in "nude" mice. JExp Med. 1971;134(3 Pt 1):681-692.

22. Radha Rama Devi A, Panday NN, Naushad SM. FOXN1 Italian founder mutation in Indian family: Implications in prenatal diagnosis. Gene. 2017;627:222-225.

23. Chou J, Massaad MJ, Wakim RH, Bainter W, Dbaibo G, Geha RS. A novel mutation in FOXN1 resulting in SCID: a case report and literature review. Clin Immunol. 2014;155(1):30-32.

24. Vigliano I, et al. FOXN1 mutation abrogates prenatal T-cell development in humans. JMed Genet. 2011;48(6):413-416.
25. Pignata C, et al. Congenital Alopecia and nail dystrophy associated with severe functional T-cell immunodeficiency in two sibs. Am JMed Genet. 1996;65(2):167-170.

26. Li B, Li J, Devlin BH, Markert ML. Thymic microenvironment reconstitution after postnatal human thymus transplantation. Clin Immunol. 2011;140(3):244-259.

27. Abitbol M, Bossé P, Thomas A, Tiret L. A deletion in FOXN1 is associated with a syndrome characterized by congenital hypotrichosis and short life expectancy in Birman cats. PLoS One. 2015;10(3):e0120668.

28. Romano R, et al. FOXN1: A master regulator gene of thymic epithelial development program. Front Immunol. 2013;4:187.

29. Zweier C, Sticht H, Aydin-Yaylagül I, Campbell CE, Rauch A. Human TBX1 missense mutations cause gain of function resulting in the same phenotype as 22q11.2 deletions. Am J Hum Genet. 2007;80(3):510-517.

30. Yagi H, et al. Role of TBX1 in human del22q11.2 syndrome. Lancet. 2003;362(9393):1366-1373.

31. Paganini I, et al. A novel PAX1 null homozygous mutation in autosomal recessive otofaciocervical syndrome associated with severe combined immunodeficiency. Clin Genet. 2017;92(6):664-668.

32. Pohl E, et al. A hypofunctional PAX1 mutation causes autosomal recessively inherited otofaciocervical syndrome. Hum Genet. 2013;132(11):1311-1320.

33. Gennery AR, et al. Mutations in CHD7 in patients with CHARGE syndrome cause T-B + natural killer cell + severe combined immune deficiency and may cause Omenn-like syndrome. Clin Exp Immunol. 2008;153(1):75-80.

34. Liu N, et al. Functional variants in TBX2 are associated with a syndromic cardiovascular and skeletal developmental disorder. Hum Mol Genet. 2018;27(14):2454-2465.

35. Morsheimer M, Brown Whitehorn TF, Heimall J, Sullivan KE. The immune deficiency of chromosome 22q11.2 deletion syndrome. Am JMed Genet A. 2017;173(9):2366-2372. 
36. Kwan A, et al. Newborn screening for severe combined immunodeficiency in 11 screening programs in the United States. JAMA. 2014;312(7):729-738.

37. Shearer WT, et al. Lymphocyte subsets in healthy children from birth through 18 years of age: the Pediatric AIDS Clinical Trials Group P1009 study. JAllergy Clin Immunol. 2003;112(5):973-980.

38. Cordier AC, Haumont SM. Development of thymus, parathyroids, and ultimo-branchial bodies in NMRI and nude mice. Am J Anat. 1980;157(3):227-263.

39. Uddin MM, et al. Foxn1- $\beta 5$ t transcriptional axis controls $\mathrm{CD}^{+} \mathrm{T}$-cell production in the thymus. Nat Commun. 2017;8:14419.

40. Johns SA, Soullier S, Rashbass P, Cunliffe VT. Foxn1 is required for tissue assembly and desmosomal cadherin expression in the hair shaft. Dev Dyn. 2005;232(4):1062-1068.

41. Farley AM, et al. Dynamics of thymus organogenesis and colonization in early human development. Development. 2013;140(9):2015-2026.

42. Chen L, Xiao S, Manley NR. Foxn1 is required to maintain the postnatal thymic microenvironment in a dosage-sensitive manner. Blood. 2009;113(3):567-574.

43. Zook EC, et al. Overexpression of Foxn1 attenuates age-associated thymic involution and prevents the expansion of peripheral CD 4 memory T cells. Blood. 2011;118(22):5723-5731.
44. Sun L, Guo J, Brown R, Amagai T, Zhao Y, Su DM. Declining expression of a single epithelial cell-autonomous gene accelerates age-related thymic involution. Aging Cell. 2010;9(3):347-357.

45. Bredenkamp N, Nowell CS, Blackburn CC. Regeneration of the aged thymus by a single transcription factor. Development. 2014;141(8):1627-1637.

46. Potter CS, et al. The nude mutant gene Foxn1 is a HOXC13 regulatory target during hair follicle and nail differentiation. J Invest Dermatol. 2011;131(4):828-837.

47. Su DM, Navarre S, Oh WJ, Condie BG, Manley NR. A domain of Foxn1 required for crosstalkdependent thymic epithelial cell differentiation. Nat Immunol. 2003;4(11):1128-1135.

48. Gui J, Mustachio LM, Su DM, Craig RW. Thymus size and age-related thymic involution: early programming, sexual dimorphism, progenitors and stroma. Aging Dis. 2012;3(3):280-290.

49. Haynes BF, Markert ML, Sempowski GD, Patel DD, Hale LP. The role of the thymus in immune reconstitution in aging, bone marrow transplantation, and HIV-1 infection. Annu Rev Immunol. 2000;18:529-560.

50. Belkaya S, et al. Dynamic modulation of thymic microRNAs in response to stress. PLoS One. 2011;6(11):e27580.

51. Markert ML, et al. Review of 54 patients with complete DiGeorge anomaly enrolled in protocols for thymus transplantation: out- come of 44 consecutive transplants. Blood. 2007;109(10):4539-4547.

52. Markert ML, et al. Thymus transplantation in complete DiGeorge syndrome: immunologic and safety evaluations in 12 patients. Blood. 2003;102(3):1121-1130.

53. Belkaya S, Murray SE, Eitson JL, de la Morena MT, Forman JA, van Oers NS. Transgenic expression of microRNA- 185 causes a developmental arrest of $\mathrm{T}$ cells by targeting multiple genes including Mzb1. J Biol Chem. 2013;288(42):30752-30762.

54. Hoover AR, et al. MicroRNA-205 maintains T cell development following stress by regulating forkhead box N1 and selected chemokines. J Biol Chem. 2016;291(44):23237-23247.

55. Williams KM, Mella H, Lucas PJ, Williams JA, Telford W, Gress RE. Single cell analysis of complex thymus stromal cell populations: rapid thymic epithelia preparation characterizes radiation injury. Clin Transl Sci. 2009;2(4):279-285.

56. Du Q, et al. MIR205HG is a long noncoding RNA that regulates growth hormone and prolactin production in the anterior pituitary. Dev Cell. 2019;49(4):618-631.e5.

57. DeFord-Watts LM, et al. The CD3 zeta subunit contains a phosphoinositide-binding motif that is required for the stable accumulation of TCRCD3 complex at the immunological synapse. JImmunol. 2011;186(12):6839-6847. 